\title{
Aspects of Integrability in Self-Dual Einstein Metrics and Related Equations
}

By

\author{
Kanehisa TAKASAKI*
}

\begin{abstract}
Abstrace
The nonlinear system describing self-dual Einstein metrics and its generalizations are discussed from the point of view of integrability. It is shown that these nonlinear systems share a variety of remarkable features (such as the existence of a linear scattering problem, a group-theoretical solution technique similar to the Riemann-Hilbert problem, and a geometric interpretation as dynamical motion in an infinite dimensional Grassmann manifold) with nonlinear integrable systems known until now. Differences of the relevant group-theoretical structures between these two classes of nonlinear systems are also pointed out. These results lead to the conclusion that the nonlinear systems in question do form a new class of nonlinear integrable systems.
\end{abstract}

\section{Introduction}

Along with the developments in the last decade the notion of "complete integrability," or "integrability" for brevity, has become considerably familiar to us. Some of its mathematical issues, nevertheless, seem to remain to be fully solved. A basic question in this context is, for example, how far the notion of nonlinear integrable systems can be really extended. In the beginning of this field just a few examples, including the celebrated Korteweg-de Vries equation, were known as nonlinear integrable systems, but after the discovery and progress of a number of techniques we now have an enormous list of "soliton equations" [1-4]. There are also attempts of quantization of these equations [5]. Further, some of these techniques later turned out to be also applicable to the self-dual Yang-Mills equations [6-10]. Recently their higher dimensional [11] and supersymmetric [12-14] analogues were investigated along the same lines. The frontier of what should be called nonlinear integrable

Received May 13, 1986.

* Research Insitute for Mathematical Sciences, Kyoto University, Kyoto 606, Japan. 
systems thus has been (and, perhaps, is still) expanding continuously.

The purpose of this paper is to show that the system of nonlinear equations defining self-dual Einstein metrics, which we call the self-dual Einstein equations, share various remarkable features with nonlinear integrable systems known until now. This means that the self-dual Einstein equations should be considered a sort of nonlinear integrable system. Such a point of view is in fact not very new. Rather, in view of the fact that the integrability of the self-dual Yang-Mills equations is a consequence of their twistor-theoretical interpretation [15], it would be quite natural to expect a similar situation for the self-dual Einstein equations, because the latter also admit a twistorial description due to Penrose [16]. The recent work of Boyer and Plebanski $[17,18]$ seems to be indeed based, at least partly, on such an idea. This paper is intended to present a more detailed analysis on this issue.

Of course the notion of integrability includes in general a variety of contents, but this paper deals with the self-dual Einstein equations from the following three aspects (which are in fact closely connected with each other):

i) The existence of a linear "scattering" problem whose integrability conditions in the sense of Frobenius agree with the nonlinear system in question.

ii) A group-theoretical description of solutions with the aid of the Riemann-Hilbert problem or of its appropriate analogue.

iii) A geometric interpretation of the nonlinear system as some dynamical motion in a symmetric space (in particular, in an infinite dimensional Grassmann manifold).

Detailed discussions on these aspects will be presented in the subsequent sections (see $\$ 2$ for (i), $\$ 4$ for (ii) and $\S 5$ for (iii)). It would be however worth mentioning here in advance that group-theoretical structures relevant to the self-dual Einstein equations are somewhat distinct from (though seemingly fairly similar to) the case of more classical examples of nonlinear integrable systems. For example, as already pointed by Boyer and Plebanski [18], an infinite dimensional Lie algebra characteristic of the present case takes the form of a tensor product $\gamma \otimes C\left[\lambda, \lambda^{-1}\right]$ where $\lambda$ is a parameter and $\gamma$ a Lie algebra of vector fields which is infinite dimensional in itself; on the other hand for soliton equations [2-4] and the self-dual or supersymmetric Yang-Mills equations $[10,13,14]$ the same role is played by Kac-Moody-type Lie algebras, which are typically written $\mathfrak{g} \otimes \mathbb{C}\left[\lambda, \lambda^{-1}\right]$ with $\mathfrak{g}$ finite dimensional Lie algebras. For this and some other reasons to be made clear in the subsequent sections, it 
seems very natural to recognize the self-dual Einstein equations as an essentially new type of nonlinear integrable system. Besides, this is by no means an isolated example; it will be indeed shown in $\$ 3$ that there is a broad class of nonlinear systems that should be considered "integrable" in the same sense.

\section{§1. Potentials for Self-Dual Eimstein Metries}

\subsection{Self-Dudl Eimstein Metries}

Let $d s^{2}=g_{\mu \nu}(x) d x^{\mu} d x^{v}$ denote a four-dimensional Riemannian metric with coordinates $x=\left(x^{1}, x^{2}, x^{3}, x^{4}\right)$, and $R_{\alpha \beta \mu \nu}$ the components of the Riemann curvature forms. The metric $d s^{2}$ is said to be a self-dual Einstein metric if the Riemann curvature forms are self-dual with respect to the Hodge *-operator, i.e.

$$
* R_{\alpha \beta \mu \nu} d x^{\mu} \wedge d x^{\nu}=R_{\alpha \beta \mu \nu} d x^{\mu} \wedge d x^{\nu} .
$$

The usual (vacuum) Einstein equations $R_{\mu \nu}=0$ automatically follow from $\mathbb{E}$. (1.1) (see, for example, $[19,20]$ ), thus self-dual Einstein metrics form part of Einstein metrics. We call Eqs. (1.1) the self-dual Einstein equations.

Since the self-dual Einstein equations, as well as the Einstein equations, include only rational functions of derivatives of the metric components $g_{\mu \nu}(x)$, one may readily replace the real coordinates $x=\left(x^{1}, x^{2}, x^{3}, x^{4}\right)$ by complex ones $z=\left(z^{1}, z^{2}, z^{3}, z^{4}\right)$ and consider these equations in complex domains. In this paper we mainly deal with these "complexified" equations, calling them also the self-dual Einstein equations.

\subsection{Nunl-Tetrad Formalism}

Plebanski [20] pointed out that null-tetrad formalism is very useful for the study of self-dual Einstein metrics. In this formalism a metric is written

$$
d s^{2}=2 e^{1} e^{2}+2 e^{3} e^{4}=2 \operatorname{det}\left(\begin{array}{rr}
e^{3} & e^{1} \\
-e^{2} & e^{4}
\end{array}\right)
$$

where $e^{1}, \ldots, e^{4}$ denote a tetrad (vierbein), i.e. linearly independent 1 -forms $e^{a}=e_{\mu}^{a} d z^{\mu}$. Writing the metric as above is not unique; there remains the following arbitrariness: 


$$
\left(\begin{array}{rr}
e^{3} & e^{1} \\
-e^{2} & e^{4}
\end{array}\right) \longrightarrow l\left(\begin{array}{rr}
e^{3} & e^{1} \\
-e^{2} & e^{4}
\end{array}\right) l^{\prime}
$$

where $l=l(z)$ and $l^{\prime}=l^{\prime}(z)$ are $\operatorname{SL}(2, \mathbb{C})$-valued arbitrary functions. We call these transformations induced by $l$ and $l$, respectively, left and right $\operatorname{SL}(2, \mathbb{C})$ gauge transformations of the tetrad.

Main results of Plebanski [20] can be summarized as follows. First, by choosing an appropriate right $\operatorname{SL}(2, \mathbb{C})$ gauge transformation the self-dual Einstein equations for metric (1.2) can be reduced to the exterior differential equations

$$
d\left(e^{2} \wedge e^{3}\right)=0, \quad d\left(e^{3} \wedge e^{4}-e^{1} \wedge e^{2}\right)=0, \quad d\left(e^{1} \wedge e^{4}\right)=0 .
$$

By introducing a new parameter $\lambda$, they can be written more compactly as:

$$
d\left(\left(e^{3}+\lambda e^{1}\right) \wedge\left(-e^{2}+\lambda e^{4}\right)\right)=0 \quad(\lambda \in \mathbb{C}) .
$$

Second, performing further a left $\operatorname{SL}(2, \mathbb{C})$ gauge transformation one can bring the tetrad into simple canonical forms. Actually Plebanski presented two sorts of such canonical forms, one of which takes the following form:

$$
\begin{aligned}
e^{3}=d x-\Theta_{x y} d p-\Theta_{y y} d q, & e^{1}=d p, \\
-e^{2}=d y+\Theta_{x x} d p+\Theta_{x y} d q, & e^{4}=d q,
\end{aligned}
$$

where $(p, q, x, y)$ are appropriate coordinates and $\Theta=\Theta(p, q, x, y)$ is a solution of the equation (second heavenly equation)

$$
\Theta_{x q}-\Theta_{y p}+\Theta_{x x} \Theta_{y y}-\Theta_{x y}^{2}=0,
$$

whereas the indices to $\Theta$ as usual denote the differentiation with respect to the variables indicated therein. It should be also noted that Eqs. (1.4) are forminvariant under left $\operatorname{SL}(2, \mathbb{C})$ gauge transformations.

\subsection{Definition of $u$-Potentials}

What play central roles throughout this paper are potentials $u_{n}^{\alpha}=u_{n}^{\alpha}(z)$ and $\hat{u}_{n}^{\alpha}=\hat{u}_{n}^{\alpha}(z) \quad(n=1,0,-1, \ldots, \alpha=1,2)$ and their generating functions (which we call, for brevity, $u$-potentials)

$$
u^{\alpha}=\sum_{n=-\infty}^{1} u_{n}^{\alpha} \lambda^{n}, \hat{u}^{\alpha}=\sum_{n=0}^{\infty} \hat{u}_{n}^{\alpha} \lambda^{n} \quad(\alpha=1,2)
$$

to be defined respectively as a solution of the exterior differential equations

$$
d u^{1} \wedge d u^{2}=\left(e^{3}+\lambda e^{1}\right) \wedge\left(-e^{2}+\lambda e^{4}\right)
$$




$$
d \hat{u}^{1} \wedge d \hat{u}^{2}=\left(e^{3}+\lambda e^{1}\right) \wedge\left(-e^{2}+\lambda e^{4}\right),
$$

where (and also throughout this paper) $\lambda$ denotes a parameter moving in the Riemann sphere $\mathbb{P}^{1}$, and $d$ the total differentiation with respeci to space-time coordinates alone (i.e. $d \lambda=0$ ). In general these potentials $u^{\alpha}$ and $\hat{u}^{\alpha}$ are required to be just formal Laurent series of $\lambda$ (though in applications to various actual solutions they can be chosen convergent ones; see §4). The existence of such potentials evidently implies that the tetrad satisfies Eqs. (1.4), because the left side of Eqs. (1.8) and (1.9) are always closed forms. What is important is that the converse is also true:

Proposition (1.10)。 For a given tetrad such potentials $u^{\alpha}$ and $\hat{u}^{\alpha}$ do exist if and only if Eqs. (1.4) are satisfied.

In other words Eqs. (1.4) are exactly the integrability conditions for Eqs. (1.8) and (1.9) to have a solution (not unique).

There are various ways to verify the above basic fact. Newman et al. argued a construction of such potentials in [21], which seems to be an earliest attempt that introduced equations like (1.8) and (1.9) into the study of the self-dual Einstein equations. Boyer and Plebanski $[17,18]$ presented another construction exploiting a geometric formalism. (In fact, what Boyer and Plebanski called "potentials" are different from ours; they used this word to mean another sort of potential functions including the function $\Theta$.) According to their argument, our $u$-potentials can be identified with coordinate components of a section of a certain infinite dimensional vector bundle (on the $z$-space) endowed with a sort of symplectic structure; the problem of solving Eqs. (1.8) and (1.9) then becomes equivalent to finding an isotropic section of this vector bundle. A common characteristic of these two constructions is to reduce the problem into solving an infinite system of equations which can be obtained by expanding the both sides of Eqs. (1.8) and (1.9) into Laurent series of $\lambda$. There, however, is another method that enables one to construct the $u$-potentials more directly. This method is an application of Darboux's theorem [22] on the canonical forms of exterior differential 2-forms. This theorem shows that if a 2-form $\omega$ on a finite dimensional manifold is closed (i.e. $d \omega=0$ ) and for some integer $r(r \geq 1) \omega \wedge \cdots \wedge \omega(r$-fold $) \neq 0$ and $\omega \wedge \cdots \wedge \omega(r+1$-fold $)=0$, then at any point there are a neighborhood of this point and functions $P_{1}, \ldots, P_{r}, Q_{1}, \ldots$, $Q_{r}$ defined therein such that $d P_{1} \wedge \cdots \wedge d P_{r} \wedge d Q_{1} \wedge \cdots \wedge d Q_{r} \neq 0$ and $\omega=\sum_{i=1}^{r} d P_{i}$ $\wedge d Q_{i}$. If the manifold is even, say $2 n$, dimensional and $r=n$, then this is a 
well known result in symplectic geometry; therefore following the terminology of symplectic geometry, let us call the functions $P_{1}, \ldots, P_{r}, Q_{1}, \ldots, Q_{r}$ canonical variables. Darboux's theorem can be extended to the case where the 2-form includes some additional parameters, and such a modified form of Darboux's theorem can be applied to the construction of the $u$-potentials. Indeed, the right side of Eqs. (1.8) and (1.9) is a closed 2-form with a parameter $\lambda$, provided the tetrad satisfies Eqs. (1.4). Furthermore, for this 2 -form the integer $r$ is equal to one. Therefore by virtue of Darboux's theorem one obtains a pair of canonical variables, which are exactly the $u$-potentials in question. Of course one should be careful here about the domains in the Riemann sphere in which the parameter $\lambda$ moves. Namely, the two sorts of potentials $u^{\alpha}$ and $\hat{u}^{\alpha}$ both can be obtained as canonical variables in the canonical form of the 2 -form $\left(e^{1}+\lambda e^{3}\right) \wedge\left(-e^{2}+\lambda e^{4}\right)$, but the domains in which the parameter $\lambda$ is supposed to move are distinct; for the former ones the domains is a neighborhood of $\lambda=\infty$, whereas for the latter it is a neighborhood of $\lambda=0$.

The notion of $u$-potentials forms a central theme of this paper; a variety of roles to be palyed by them will be described in detail in the following sections. It is however worth noting here that these potentials are also the most basic ingredients of the curved twistor construction of self-dual Einstein metrics due to Penrose [16]. For details, see $§ 4$; as we shall argue therein, these potentials can be regarded as representing a family of holomorphic curves in a curved twistor space.

\section{4. u-Potentials and Canonical Form of Tetrad}

We here show that the coordinates $(p, q, x, y)$ can be identified with the leading and next-to-leading coefficients of the Laurent expansion of the potentials $u^{1}$ and $u^{2}$; this is a basic property of $u$-potentials, which sometimes enables us to save calculations to a great extent. To see the above fact, let us first note that Eq. (1.8) is equivalent to the system of equations

$$
\begin{aligned}
& \omega^{(2)}=e^{1} \wedge e^{4}, \\
& \omega^{(1)}=e^{3} \wedge e^{4}-e^{1} \wedge e^{2}, \\
& \omega^{(0)}=e^{2} \wedge e^{3}, \\
& \omega^{(n)}=0 \quad(n<0),
\end{aligned}
$$

where $\omega^{(n)}$ denotes the 2-form $\omega^{(n)} \equiv \sum_{m=n-1}^{1} d u_{m}^{1} \wedge d u_{n-m}^{2}$. From Eqs. $(1.10)_{2}$ and $(1.10)_{0}$ one finds that 


$$
d u_{1}^{1} \wedge d u_{1}^{2} \wedge d u_{0}^{1} \wedge d u_{0}^{2}=\omega^{(2)} \wedge \omega^{(0)}=e^{1} \wedge e^{2} \wedge e^{3} \wedge e^{4} \neq 0
$$

therefore one may take $\left(u_{1}^{1}, u_{1}^{2}, u_{0}^{1}, u_{0}^{2}\right)$ as new space-time coordinates. We now prove:

Proposition (1.12). Take $(p, q, x, y) \equiv\left(u_{1}^{1}, u_{1}^{2}, u_{0}^{1}, u_{0}^{2}\right)$ as space-time coordinates and regard the other Laurent coefficients as functions of $(p, q, x, y)$. Then there are an $\operatorname{SL}(2, \mathbb{C})$-valued function $l=\left(l_{\alpha}^{\beta}(p, q, x, y)\right)$ and a scalar function $\Theta \equiv \Theta(p, q, x, y)$ satisfying the following equations:

$$
\begin{gathered}
\left(\begin{array}{rr}
e^{3} & e^{1} \\
-e^{2} & e^{4}
\end{array}\right)=l\left(\begin{array}{ll}
d x-\Theta_{x y} d p-\Theta_{y y} d q & d p \\
d y+\Theta_{x x} d p+\Theta_{x y} d q & d p
\end{array}\right), \\
\Theta_{x q}-\Theta_{y p}+\Theta_{x x} \Theta_{y y}-\Theta_{x y}^{2}=0 \\
u_{-1}^{1}=\Theta_{y}, \quad u_{-1}^{2}=-\Theta_{x} .
\end{gathered}
$$

Proof. From Eqs. $(1.10)_{1}$ and $(1.10)_{0}$

$$
\begin{aligned}
& \omega^{(1)} \wedge \omega^{(0)}=\left(e^{3} \wedge e^{4}-e^{1} \wedge e^{2}\right) \wedge e^{2} \wedge e^{3}=0, \\
& \omega^{(0)} \wedge \omega^{(0)}=e^{2} \wedge e^{3} \wedge e^{2} \wedge e^{3}=0,
\end{aligned}
$$

whereas in terms of the coordinates $(p, q, x, y)$ one can calculate these 2-forms as:

$$
\begin{aligned}
\omega^{(1)} \wedge \omega^{(0)}=-d p \wedge d q \wedge d x & \wedge d y\left(\frac{\partial u_{-1}^{1}}{\partial x}+\frac{\partial u_{-1}^{2}}{\partial y}\right) \\
\omega^{(0)} \wedge \omega^{(0)}=2 d p \wedge d q \wedge d x \wedge d y & \left(\frac{\partial u_{-1}^{1}}{\partial p}+\frac{\partial u_{-1}^{2}}{\partial q}\right. \\
& \left.+\frac{\partial u_{-1}^{2}}{\partial x} \frac{\partial u_{-1}^{1}}{\partial y}-\frac{\partial u_{-1}^{2}}{\partial y}-\frac{\partial u_{-1}^{1}}{\partial x}\right),
\end{aligned}
$$

therefore one obtains the equations

$$
\begin{gathered}
\frac{\partial u_{-1}^{1}}{\partial x}+\frac{\partial u_{-1}^{2}}{\partial y}=0 \\
\frac{\partial u_{-1}^{1}}{\partial p}+\frac{\partial u_{-1}^{2}}{\partial q}+\frac{\partial u_{-1}^{2}}{\partial x} \frac{\partial u_{-1}^{1}}{\partial y}-\frac{\partial n_{-1}^{2}}{\partial y} \frac{\partial u_{-1}^{1}}{\partial x}=0 .
\end{gathered}
$$

Eq. (1.13a) ensures the existence of a function $\Theta$ that satisfies Eq. (1.12c), and inserting this expression of $u_{-1}^{1}$ and $u_{-1}^{2}$ into Eq. (1.13b) one obtains Eq. (1.12b). Thus what remains is to prove the existence of a left $\operatorname{SL}(2, \mathbb{C})$ gauge transformation that connects the tetrad $\left(e^{1}, \ldots, e^{4}\right)$ with the following one:

$$
\begin{array}{rlr}
\tilde{e}^{3}=d x-\Theta_{x y} d p-\Theta_{y y} d q, & \tilde{e}^{1}=d p, \\
-\tilde{e}^{2}=d y+\Theta_{x x} d p+\Theta_{x y} d q, & \tilde{e}^{4}=d q .
\end{array}
$$


To check this, let us note the equations

$$
\begin{aligned}
& e^{1} \wedge e^{4}=\omega^{(2)}=\tilde{e}^{1} \wedge \tilde{e}^{4}, \\
& e^{3} \wedge e^{4}-e^{1} \wedge e^{2}=\omega^{(1)}=\tilde{e}^{3} \wedge \tilde{e}^{4}-\tilde{e}^{1} \wedge \tilde{e}^{2}, \\
& e^{2} \wedge e^{3}=\omega^{(0)}=\tilde{e}^{1} \wedge \tilde{e}^{4},
\end{aligned}
$$

which follow from Eqs. (1.10) and (1.13). It is then a simple excercise of linear algebra to show from the last equations that there is certainly a left $\operatorname{SL}(2, \mathbb{C})$ gauge transformation as such. This completes the proof of the proposition.

\section{§ 2. Linear Scattering Problem for Self-Dual Einstein Metrics}

\subsection{Derivation of Linear Problem}

In the preceding section we introduced the notion of $u$-potentials. We call these new dependent variables "potentials" (or "quasi-potentials", according to the terminology of Estabrook and Wahlquist [23]) because they are defined as solutions of differential equations, i.e. (1.8) and (1.9), whose integrability conditions coincide with the equations in question, i.e. Eqs. (1.4). Eqs. (1.8) and (1.9) are however neither linear nor quasi-linear, thus the situation is seemingly very different from various (quasi-) potentials (in particular, "wave functions" in linear scattering problems) to be defined for nonlinear integrable systems known until now [1-14]. Thus naturally occurs a question, whether or not there is a linear problem which characterizes our $u$-potentials. The conclusion we here derive is that such a linear problem certainly exists if one admits a nonlinear constraint to be added to the relevant linear equations.

In order to give a precise statement, we intorudce the notion of dual (or inverse) tetrad. The dual tetrad of a given tetrad $\left(e^{1}, \ldots, e^{4}\right)$ is by definition the tangent frame $\left(\partial_{1}, \ldots, \partial_{4}\right)$ of vector fields to be determined by the equations

$$
\left\langle e^{a}, \partial_{b}\right\rangle=\delta_{b}^{a} \quad \text { for } \quad a, b=1, \ldots, 4,
$$

where $\langle$,$\rangle denotes the natural inner product of 1$-forms and vector fields, and $\delta_{b}^{a}$ the Kronecker delta. A basic property of the dual tetrad is that the following formula holds for any function $h=h(z)$ :

$$
d h=\sum_{a=1}^{4}\left(\partial_{a} h\right) e^{a} .
$$

The linear problem in question can be obtained by rewriting Eqs. (1.8) and 
(1.9) using the dual tetrad of the tetrad included therein. Actually it has two equivalent representations, one of which takes the following form:

Proposition (2.3). Let $\left(\partial_{1}, \ldots, \partial_{4}\right)$ denote the dual tetrad of the tetrad $\left(e^{1}, \ldots, e^{4}\right)$. Then Eq. (1.8) is equivalent to the system of equations

$$
\begin{gathered}
\left(\begin{array}{l}
d u^{1} \\
d u^{2}
\end{array}\right)=\left(\begin{array}{ll}
\partial_{3} u^{1} & -\partial_{2} u^{1} \\
\partial_{3} u^{2} & -\partial_{2} u^{2}
\end{array}\right)\left(\begin{array}{c}
e^{3}+\lambda e^{1} \\
-e^{2}+\lambda e^{4}
\end{array}\right), \\
\left(\partial_{2} u^{1}\right)\left(\partial_{3} u^{2}\right)-\left(\partial_{3} u^{1}\right)\left(\partial_{2} u^{2}\right)=1 .
\end{gathered}
$$

Similarly, Eq. (1.9) is equivalent to the same equations in which $u^{1}$ and $u^{2}$ are replaced by $\hat{u}^{1}$ and $\hat{u}^{2}$.

Proof. It is obvious that Eq. (1.8) follows from Eqs. (2.3), so let us check the converse. In view of (2.2) one can expand the 2 -form $d u^{1} \wedge d u^{2}$ as:

$$
\begin{aligned}
& d u^{1} \wedge d u^{2}=\sum_{a<b} d u^{1} \wedge d u^{2}\left(\partial_{a}, \partial_{b}\right), \text { where } \\
& d u^{1} \wedge d u^{2}\left(\partial_{a}, \partial_{b}\right) \equiv\left(\partial_{a} u^{1}\right)\left(\partial_{b} u^{2}\right)-\left(\partial_{a} u^{2}\right)\left(\partial_{b} u^{1}\right),
\end{aligned}
$$

therefore equating the coefficients of $e^{a} \wedge e^{b}$ in Eq. (1.8) one obtains:

$$
\begin{aligned}
& d u^{1} \wedge d u^{2}\left(\partial_{1}, \partial_{2}\right)=-\lambda, \quad d u^{1} \wedge d u^{2}\left(\partial_{1}, \partial_{3}\right)=0 \\
& d u^{1} \wedge d u^{2}\left(\partial_{1}, \partial_{4}\right)=\lambda^{2}, \quad d u^{1} \wedge d u^{2}\left(\partial_{2}, \partial_{3}\right)=1 \\
& d u^{1} \wedge d u^{2}\left(\partial_{2}, \partial_{4}\right)=0, \quad d u^{1} \wedge d u^{2}\left(\partial_{3}, \partial_{4}\right)=\lambda
\end{aligned}
$$

The fourth equation in (2.5) is exactly Eq. (2.3b). This in particular implies:

$$
\left(\begin{array}{cc}
\partial_{3} u^{1} & -\partial_{2} u^{1} \\
\partial_{3} u^{2} & -\partial_{2} u^{2}
\end{array}\right)^{-1}\left(\begin{array}{l}
d u^{1} \\
d u^{2}
\end{array}\right)=\left(\begin{array}{l}
-\left(\partial_{2} u^{2}\right) d u^{1}+\left(\partial_{2} u^{1}\right) d u^{2} \\
-\left(\partial_{3} u^{2}\right) d u^{1}+\left(\partial_{3} u^{1}\right) d u^{2}
\end{array}\right)
$$

and calculating the right side further using Eqs. (2.5), one finds:

$$
=\left(\begin{array}{r}
e^{3}+\lambda e^{1} \\
-e^{2}+\lambda e^{4}
\end{array}\right)
$$

thus it turns out that Eq. (2.3a), too, follows from Eq. (1.8). The latter half of the proposition can be checked the same way. This completes the proof of the proposition.

We now give another representation of the linear problem:

Proposition (2.6). Let $\left(\partial_{1}, \ldots, \partial_{4}\right)$ denote the dual tetrad of the tetrad $\left(e^{1}, \ldots, e^{4}\right)$. Then Eq. (1.8) is equivalent to the system of equations

$$
\begin{aligned}
& \left(-\lambda \partial_{3}+\partial_{1}\right) u=0, \quad\left(\lambda \partial_{2}+\partial_{4}\right) u=0 \quad\left(u=u^{1}, u^{2}\right), \\
& \left(\partial_{2} u^{1}\right)\left(\partial_{3} u^{2}\right)-\left(\partial_{3} u^{1}\right)\left(\partial_{2} u^{2}\right)=1 .
\end{aligned}
$$


Similarly, Eq. (1.9) is equivalent to the same equations in which $u^{1}$ and $u^{2}$ are replaced by $\hat{u}^{1}$ and $\hat{u}^{2}$.

Proof. One may rewrite Eq. (2.3a) into the following form:

$$
d u=\left(\lambda \partial_{3} u\right) e^{1}+\left(\partial_{2} u\right) e^{2}+\left(\partial_{3} u\right) e^{3}-\left(\lambda \partial_{2} u\right) e^{4} \quad\left(u=u^{1}, u^{2}\right) .
$$

On the other hand by virtue of (2.2) the 1 -form $d u$ may be in general written

$$
d u=\left(\partial_{1} u\right) e^{1}+\left(\partial_{2} u\right) e^{2}+\left(\partial_{3} u\right) e^{3}+\left(\partial_{4} u\right) e^{4} .
$$

Therefore equating the coefficients of $e^{1}$ and $e^{4}$ one readily finds that Eqs. (2.6) are equivalent to Eqs. (2.3) and, consequently, to Eq. (1.8). The latter half of the proposition can be verified the same way. This completes the proof of the proposition.

The last system of equations, (2.6), is what we have sought for. This system indeed takes the form of a linear problem coupled with a nonlinear constraint, and the $u$-potentials are characterized by this system.

\subsection{Symplectic Structure in Linear Problem}

We here show that a sort of symplectic structure is underlying linear problem (2.6) and explains the meaning of nonlinear constraint (2.6b). This symplectic structure manifests itself when, as Proposition (1.12) ensures, the tetrad is cast into the second canonical form of Plebanski, (1.6). In order to see this, we use the following lemmas which can be readily checked:

Lemma (2.7). Under a transformation of the tetrad as in (1.3) the corresponding dual tetrad changes as:

$$
\left(\begin{array}{rr}
\partial_{3} & \partial_{1} \\
-\partial_{2} & \partial_{4}
\end{array}\right) \longrightarrow{ }^{t-1}\left(\begin{array}{rr}
\partial_{3} & \partial_{1} \\
-\partial_{2} & \partial_{4}
\end{array}\right) l^{t-1}
$$

Lemma (2.8)。 The dual tetrad of tetrad (1.6) is:

$$
\left(\begin{array}{cc}
\partial_{3} & \partial_{1} \\
-\partial_{2} & \partial_{4}
\end{array}\right)=\left(\begin{array}{cc}
\frac{\partial}{\partial x} & \frac{\partial}{\partial p}-\Theta_{x x} \frac{\partial}{\partial y}+\Theta_{x y} \frac{\partial}{\partial x} \\
\frac{\partial}{\partial y} & \frac{\partial}{\partial q}-\Theta_{x y} \frac{\partial}{\partial y}+\Theta_{y y} \frac{\partial}{\partial x}
\end{array}\right) .
$$

We call transformations induced by $l$ and $l^{\prime}$ as above, respectively, left and right $\mathrm{SL}(2, \mathbb{C})$ gauge transformations of the dual tetrad. Note that Eqs. (2.6) are form-invariant under left $\operatorname{SL}(2, \mathbb{C})$ gauge transformations.

Let us now reduce Eqs. (2.6) into a more explicit form by using the above 
lemmas. These lemmas, applied to the situation as shown in Proposition (1.12), show that the dual tetrad $\left(\partial_{1}, \ldots, \partial_{4}\right)$ takes the following form:

$$
\left(\begin{array}{cc}
\partial_{3} & \partial_{1} \\
-\partial_{2} & \partial_{4}
\end{array}\right)={ }^{t} l^{-1}\left(\begin{array}{cc}
\frac{\partial}{\partial x} & \frac{\partial}{\partial p}-\Theta_{x x} \frac{\partial}{\partial y}+\Theta_{x y} \frac{\partial}{\partial x} \\
\frac{\partial}{\partial y} & \frac{\partial}{\partial q}-\Theta_{x y} \frac{\partial}{\partial y}+\Theta_{y y} \frac{\partial}{\partial x}
\end{array}\right) .
$$

In view of the invariance property of linear problem (2.6) mentioned above, it finally turns out that the linear problem can always be set into the following form:

$$
\begin{aligned}
& \left(-\lambda \frac{\partial}{\partial x}+\frac{\partial}{\partial p}-\Theta_{x x} \frac{\partial}{\partial y}+\Theta_{x y} \frac{\partial}{\partial x}\right) u=0, \\
& \left(-\lambda \frac{\partial}{\partial y}+\frac{\partial}{\partial q}-\Theta_{x y} \frac{\partial}{\partial y}+\Theta_{y y} \frac{\partial}{\partial x}\right) u=0 \quad\left(u=u^{1}, u^{2}\right), \\
& \frac{\partial\left(u^{1}, u^{2}\right)}{\partial(x, y)} \equiv \frac{\partial u^{1}}{\partial x} \frac{\partial u^{2}}{\partial y}-\frac{\partial u^{1}}{\partial y} \frac{\partial u^{2}}{\partial x}=1
\end{aligned}
$$

where of course the following relations are assumed:

$$
\left(u_{1}^{1}, u_{1}^{2}, u_{0}^{1}, u_{0}^{2}\right)=(p, q, x, y), \quad u_{-1}^{1}=\Theta_{y}, \quad u_{-1}^{2}=-\Theta_{x} .
$$

It is now evident that the linear problem, written in the reduced form (2.10), is in close connection with the symplectic structure to be defined in the $(x, y)$-space with symplectic form $d x \wedge d y$. Eq. (2.10b) implies that the two dimensional map $(x, y) \rightarrow\left(u^{1}, u^{2}\right)$ is a canonical transformation with parameters $(p, q, \lambda)$; this clearly explains the meaning of the nonlinear constraint in the linear problem. In addition, the linear part (2.10a) also reflects the structure of this symplectic form; indeed, the vector fields in Eqs. (2.10a) take the form of Hamiltonian vector fields. To be more precise, we introduce the following notation:

$$
\begin{aligned}
& H_{h} \equiv \frac{\partial h}{\partial x} \frac{\partial}{\partial y}-\frac{\partial h}{\partial y} \frac{\partial}{\partial x} \quad(\text { Hamiltonian vector field }) \\
& \left\{h_{1}, h_{2}\right\} \equiv H_{h_{1}} h_{2}=\frac{\partial h_{1}}{\partial x} \frac{\partial h_{2}}{\partial y}-\frac{\partial h_{1}}{\partial y} \frac{\partial h_{2}}{\partial x} \text { (Poisson bracket) } .
\end{aligned}
$$

Then Eqs. (2.10) can be written

$$
\begin{gathered}
\left(-\lambda \frac{\partial}{\partial x}+\frac{\partial}{\partial p}-H_{\Theta_{x}}\right) u=0,\left(-\lambda \frac{\partial}{\partial y}+\frac{\partial}{\partial q}-H_{\Theta_{y}}\right) u=0 \\
\left\{u^{1}, u^{2}\right\}=1,
\end{gathered}
$$

thus giving a representation of the linear problem in which the relation to the 
above-mentioned symplectic structure is made fully manifest.

\subsection{Comparison with Other Nonlinear Integrable Systems}

We now consider the implications of the results we have thus far derived. The motivation of out present discussion was the question of whether or not the $u$-potentials can be characterized as solutions of some linear problem. As a result we have found the system of equations (2.6) which is equivalent to Eq. (1.8) and, therefore, whose integrability conditions agree exactly with Eqs. (1.4). Does this system fulfill all requirements to be a linear "scattering" problem of the self-dual Einstein equations?

One might feel this system of equations still insufficient as such a linear problem, because it includes an essentially nonlinear equation, (2.6b); however this is not correct. In fact, linear scattering problems for various nonlinear integrable systems known by now are also frequently accompanied with similar nonlinear constraints; a main role of such constraints is to derive the equations in question from more general ones as their reductions. Nonlinear constraints used for that purpose usually take the form of the condition that the unknown function (wave function) of the relevant linear problem takes values in a group. Nonlinear constraint $(2.6 \mathrm{~b})$ is evidently of the same nature; indeed, as we have remarked above, this constraint means that a certain two-dimensional map defined by the $u$-potentials takes values in the group (to be more precise, pseudogroup; see §4) of canonical transformations.

In order to make this analogy more explicit, let us attempt to compare the above linear problem for the self-dual Einstein equations with that of the self-dual Yang-Mills equations. The self-dual Yang-Mills equations in flat space-time have a "zero-curvature representation" [6-10] such as:

$$
\left[-\lambda\left(\frac{\partial}{\partial x}+A_{x}\right)+\frac{\partial}{\partial p}+A_{p},-\lambda\left(\frac{\partial}{\partial y}+A_{y}\right)+\frac{\partial}{\partial q}+A_{q}\right]=0
$$

where $(p, q, x, y)$ denote some complexified space-time coordinates and ( $A_{p}, A_{q}$, $A_{x}, A_{y}$ ) Yang-Mills gauge potentials with values in a matrix Lie algebra $g$. This equation gives a representation of the integrability conditions of the linear problem

$$
\begin{aligned}
& \left(-\lambda\left(\frac{\partial}{\partial x}+A_{x}\right)+\frac{\partial}{\partial p}+A_{p}\right) W=0, \\
& \left(-\lambda\left(\frac{\partial}{\partial y}+A_{y}\right)+\frac{\partial}{\partial q}+A_{q}\right) W=0
\end{aligned}
$$


where $W=W(p, q, x, y, \lambda)$ is a matrix-valued unknown function (wave function), usually required to take values in the Lie group $G$ of the Lie algebra $\mathfrak{g}$. The last requirement, which ensures that the gauge potentials surely take values in $\mathfrak{g}$, becomes in general a nonlinear constraint to the wave function; imagine, for example, the case for $G=\mathrm{SL}(r, \mathbb{C})$. It would be now quite clear from these observations that the linear problem for the self-dual Einstein equations has a structure very similar to that of the self-dual Yang-Mills equations.

Also significant, however, is the difference of the structure of groups relevant to the linear problems. Indeed in the case of the self-dual Yang-Mills equations, as well as other nonlinear integrable systems, the wave function takes values in a finite dimensional matrix group; whereas in the case of the self-dual Einstein equations the same role is played by the (pseudo-)group of two dimensional canonical transformations, an infinite dimensional object.

What we have viewed above is by no menas superficial. In $\S 4$ we shall once again examine this issue from a somewhat different point of view.

\section{§3. Generalizations of Self-Dual Eimstein Equations from Point of View of Imtegrability}

\subsection{Simplesế Example}

As we have viewed, the self-dual Einstein equations (to be more precise, the reduced form of Plebanski (1.4)) may be interpreted as the integrability conditions of a certain linear problem. This is one of remarkable features that the self-dual Einstein equations share with various nonlinear integrable systems known until now. Bearing the above fact in mind, in this section we seek for other examples with the same features, and attempt to find some general aspects of this class of nonlinear equations.

One of the simplest ways to obtain such an example is to consider the integrability conditions of the linear system

$$
\left(-\lambda \partial_{3}+\partial_{1}\right) u=0, \quad\left(\lambda \partial_{2}+\partial_{4}\right) u=0
$$

without any constraint. Here "integrability" means, as one can imagine from our previous arguments, that this linear system has a pair of functionally independent solutions; a more precise formulation of this statement will be given later in 3.2. As we shall see therein, the integrability conditions of the above 
linear problem in this sense take the form of a commutator relation such as:

$$
\begin{aligned}
{\left[-\lambda \partial_{3}+\partial_{1}, \lambda \partial_{2}+\partial_{4}\right] } & \\
& =\left(C_{0}+\lambda C_{1}\right)\left(-\lambda \partial_{3}+\partial_{1}\right)+\left(D_{0}+\lambda D_{1}\right)\left(\lambda \partial_{2}+\partial_{4}\right),
\end{aligned}
$$

where $C_{0}, C_{1}, D_{0}$ and $D_{1}$ are some functions independent of $\lambda$ to be determined by the frame of vector fields $\left(\partial_{1}, \ldots, \partial_{4}\right)$.

From the above expression of the integrability conditions, however, the relation to the self-dual Einstein equations is still less clear. To improve this, let us recall that there are various equivalent formulations of Frobenius' theorem [22]. What we used above is one of them that employs vector fields. Another one formulates the problem as the integrability of a Pfaffian system. In the present setting the Pfaffian system corresponding to linear system (3.1) can be written

$$
e^{3}+\lambda e^{1}=0, \quad-e^{2}+\lambda e^{4}=0,
$$

where $e^{1}, \ldots, e^{4}$ denote the 1 -forms satisfying duality relation (2.1), and $\lambda$ is considered a parameter, i.e. $d \lambda=0$. The integrability conditions of this Pfaffian system take the form of exterior differential equations such as:

$$
\begin{aligned}
& d\left(e^{3}+\lambda e^{1}\right) \wedge\left(e^{3}+\lambda e^{1}\right) \wedge\left(-e^{2}+\lambda e^{4}\right)=0, \\
& d\left(-e^{2}+\lambda e^{4}\right) \wedge\left(e^{3}+\lambda e^{1}\right) \wedge\left(-e^{2}+\lambda e^{4}\right)=0 \quad(\lambda \in \mathbb{C}),
\end{aligned}
$$

which therefore give another equivalent representation of Eq. (3.2). Once the integrability conditions are written as in (3.4), it is quite easy to see the relation to the self-dual Einstein equations. Indeed, Eqs. (1.4) imply Eqs. (3.4), but the converse is not true in general; thus one obtains a generalization of the self-dual Einstein equations in the sense mentioned at the beginning.

The above representation of the equations is also convenient to see their geometric meaning. Indeed with the aid of arguments exploited in refs. [17, $20,24]$, one can show easily that Eqs. (3.4) describe a class of conformally self-dual metrics. This however does not exhaust all conformally self-dual metrics. For the description of general conformally self-dual metrics some modification of the present setting is required; this issue will be discussed elsewhere.

We finally note that the above equations, derived as a generalization of Eqs. (1.4), also have a sort of gauge invariance. Namely, Eqs. (3.2) and (3.4) are respectively form-invariant under transformations of the vector fields and 1-forms such as: 


$$
\left(\begin{array}{rr}
e^{3} & e^{1} \\
-e^{2} & e^{4}
\end{array}\right) \longrightarrow l\left(\begin{array}{rr}
\partial^{3} & e^{1} \\
-e^{2} & e^{4}
\end{array}\right),\left(\begin{array}{rr}
\partial_{3} & \partial_{1} \\
-\partial_{2} & \partial_{4}
\end{array}\right) \longrightarrow{ }^{t-1}\left(\begin{array}{rr}
\partial_{3} & \partial_{1} \\
-\partial_{2} & \partial_{4}
\end{array}\right)
$$

where $2 \times 2$ matrices $l=\left(l_{\alpha}^{\beta}(z)\right)(\alpha, \beta=1,2)$ are simply assumed to be invertible; in other words the relevant structure group is $\operatorname{GL}(2, \mathbb{C})$ unlike the $\operatorname{SL}(2, \mathbb{C})$ for the case of Eqs. (1.4). We call the above transformations left $\mathrm{GL}(2, \mathbb{C})$ gauge transformations. As we shall see later, the 1 -forms $e^{1}, \ldots, e^{4}$ and the vector fields $\partial_{1}, \ldots, \partial_{4}$ can be reduced to a canonical form by an appropriate left $\mathrm{GL}(2, \mathbb{C})$ transformation.

\subsection{Solutions of Limear Problem and Application of Frobenins' Theorem}

We now give a precise formulation of the integrability of linear problem (3.1). If the presence of the parameter $\lambda$ may be ignored, this is just a simple application of Frobenius' thorem in a well known form, but in fact one has to take into consideration the presence of the parameter $\lambda$, which requires a more careful analysis. Bearing this in mind, we here prove:

Proposition (3.6). Given a frame $\left(\partial_{1}, \ldots, \partial_{4}\right)$ of linealy independent vector fields, linear problem (3.1) has two formal Laurent series solutions $u^{\alpha}(\alpha=1,2)$ with the expansion $u^{\alpha}=\sum_{n=-\infty}^{1} u_{n}^{\alpha} \lambda^{n}$, where $u_{n}^{\alpha}$ 's are functions of space-time coordinates alone with $d u_{1}^{1} \wedge d u_{1}^{2} \wedge d u_{0}^{1} \wedge d u_{0}^{2} \neq 0$, if and only if Eq. (3.2) is satisfied for some functions $C_{0}, C_{1}, D_{0}$, and $D_{1}$.

We first consider the "if" part. Writing $u=\sum_{n=-\infty}^{1} u_{n} \lambda^{n}$ and equating the coefficients of powers of $\lambda$ one obtains the infinite system of equations

$$
\begin{gathered}
-\partial_{3} u_{1}=0, \quad \partial_{2} u_{1}=0, \\
-\partial_{3} u_{n-1}+\partial_{1} u_{n}=0, \quad \partial_{2} u_{n-1}+\partial_{4} u_{n}=0 \quad(n=1,0,-1, \ldots),
\end{gathered}
$$

which gives an equivalent representation of linear problem (3.1). We now solve these equations successively applying Frobenius' theorem. It should be noted that this argument is almost parallel to the construction of wave functions for self-dual Yang-Mills fields due to Chau, Prasad and Sinha [9].

To start with, let us note that Eq. (3.2) splits into the three equations

$$
\begin{aligned}
& {\left[\partial_{2}, \partial_{3}\right]=-C_{1} \partial_{3}+D_{1} \partial_{2},} \\
& {\left[\partial_{1}, \partial_{2}\right]-\left[\partial_{3}, \partial_{4}\right]=-C_{0} \partial_{3}+C_{1} \partial_{1}+D_{0} \partial_{2}+D_{1} \partial_{4},} \\
& {\left[\partial_{1}, \partial_{4}\right]=C_{0} \partial_{1}+D_{0} \partial_{4} .}
\end{aligned}
$$

Since Eq. (3.8a) is satisfied, Frobenius' theorem ensures the existence of two 
solutions, $u_{1}^{1}$ and $u_{1}^{2}$, of linear system $(3.7)_{2}$ with $d u_{1}^{1} \wedge d u_{1}^{2} \neq 0$.

The construction of $u_{n}^{1}$ and $u_{n}^{2}(n=0,-1,-2, \ldots)$ can be achieved by induction. Namely, we suppose that $u_{n}^{1}, u_{n+1}^{1}, \ldots$ and $u_{n}^{2}, u_{n+1}^{2}, \ldots$ are already given as solutions of Eqs. (3.7) $)_{n+1},(3.7)_{n+2}, \ldots$, and then show that Eqs. $(3.7)_{n}$ certainly have a solution for $u_{n}=u_{n}^{1}$ and $u_{n}^{2}$, respectively. Eqs. (3.7) may be considered an inhomogeneous linear system with an unknown function $u_{n-1}$, and in view of Eq. (3.8a) Frobenius' theorem again ensures the existence of a solution provided that the integrability condition

$$
\left(C_{1} \partial_{1}+D_{1} \partial_{4}+\partial_{2} \partial_{1}+\partial_{3} \partial_{4}\right) u_{n}=0
$$

is satisfied. One can actually check, using Eqs. (3.7) $)_{n+1},(3.8 \mathrm{~b})$ and (3.8c), that $u_{n}^{1}$ and $u_{n}^{2}$ certainly satisfy the above integrability condition. Thus the induction process turns out to surely work, and yields a pair of solutions $u^{1}$ and $u^{2}$ of linear problem (3.1) with $d u_{1}^{1} \wedge d u_{1}^{2} \neq 0$.

What remains is the check of the condition $d u_{1}^{1} \wedge d u_{1}^{2} \wedge d u_{0}^{1} \wedge d u_{0}^{2} \neq 0$. This is actually an immediate consequence of the condition $d u_{1}^{1} \wedge d u_{1}^{2} \neq 0$ already fulfilled and of the following equation to be derived from $(3.7)_{1}$ and $(3.7)_{2}$ :

$$
\operatorname{det}\left(\partial_{a} u_{1}^{1}, \partial_{a} u_{1}^{2}, \partial_{a} u_{0}^{1}, \partial_{a} u_{0}^{2}\right)_{1 \leq a \leq 4}=\left[\operatorname{det}\left(\partial_{a} u_{1}^{1}, \partial_{a} u_{1}^{2}\right)_{a=1,4}\right]^{2} \text {. }
$$

Indeed, from the assumption $\partial_{1}$ and $\partial_{4}$ are in particular linearly independent, so that the right side of the above equation does not vanish because of the condition that $d u_{1}^{1} \wedge d u_{1}^{2} \neq 0$; therefore the generalized Jacobian determinant on the left side also does not vanish, and this implies $d u_{1}^{1} \wedge d u_{1}^{2} \wedge d u_{0}^{1} \wedge d u_{0}^{2} \neq 0$. This completes the proof of the "if" part of the proposition.

We next consider the "only if" part. For this purpose one may as well take $\left(u_{1}^{1}, u_{1}^{2}, u_{0}^{1}, u_{0}^{2}\right)$ as new space-time coordinates; note that this is certainly permitted because the condition $d u_{1}^{1} \wedge d u_{1}^{2} \wedge d u_{0}^{1} \wedge d u_{0}^{2} \neq 0$ is assumed. The "only if" part then readily follows from the following fact.

Proposition (3.11). Suppose that linear problem (3.1) has a pair of solutions $u^{1}$ and $u^{2}$ as shown in the statement of Proposition (3.6). Take $(p, q, x, y) \equiv\left(u_{1}^{1}, u_{1}^{2}, u_{0}^{1}, u_{0}^{2}\right)$ as space-time coordinates and regard other $u_{n}^{\alpha}$ 's as their functions. Then there is a $\mathrm{GL}(2, C)$-valued function $l=\left(l_{\alpha}^{\beta}(p, q, \mathrm{x}, y)\right)$ $(\alpha, \beta=1,2)$ satisfying the equations

$$
\left(\begin{array}{cc}
\partial_{3} & \partial_{1} \\
-\partial_{2} & \partial_{4}
\end{array}\right)=l\left(\begin{array}{cc}
\frac{\partial}{\partial x} & \frac{\partial}{\partial p}+\frac{\partial u_{-1}^{1}}{\partial x} \frac{\partial}{\partial x}+\frac{\partial u_{-1}^{2}}{\partial x} \frac{\partial}{\partial y} \\
\frac{\partial}{\partial y} & \frac{\partial}{\partial q}+\frac{\partial u_{-1}^{1}}{\partial y} \frac{\partial}{\partial x}+\frac{\partial u_{-1}^{2}}{\partial y} \frac{\partial}{\partial y}
\end{array}\right)
$$




$$
\begin{aligned}
{\left[-\lambda \frac{\partial}{\partial x}+\frac{\partial}{\partial p}+\frac{\partial u_{-1}^{1}}{\partial x} \frac{\partial}{\partial x}+\right.} & \frac{\partial u_{-1}^{2}}{\partial x} \frac{\partial}{\partial y},-\lambda \frac{\partial}{\partial y}+\frac{\partial}{\partial q} \\
& \left.+\frac{\partial u_{-1}^{1}}{\partial y} \frac{\partial}{\partial x}+\frac{\partial u_{-1}^{2}}{\partial y} \frac{\partial}{\partial y}\right]=0
\end{aligned}
$$

Proof. Eqs. (3.7) $)_{2}$ imply that the vector fields $\partial_{2}$ and $\partial_{3}$ take the form of linear combination of $\partial / \partial x$ and $\partial / \partial y$; therefore for some functions $l_{\alpha}^{\beta}(\alpha, \beta=1,2)$ $\partial_{3}=l_{1}^{1} \partial / \partial x+l_{1}^{2} \partial / \partial y$ and $-\partial_{2}=l_{2}^{1} \partial / \partial x+l_{2}^{2} \partial / \partial y$. Then inserting these formulas into Eqs. (3.7) $)_{1}$ one obtains the equation

$$
\operatorname{det}\left(l_{\alpha}^{\beta}\right)=\operatorname{det}\left(\partial_{a} u_{0}^{1}, \partial_{a} u_{0}^{2}\right)_{a=2,3}=\operatorname{det}\left(\partial_{a} u_{1}^{1}, \partial_{a} u_{1}^{2}\right)_{a=1,4},
$$

whose right side does not vanish as we have remarked above. Now let us check that the matrix $l=\left(l_{\alpha}^{\beta}\right)$ indeed fulfills all the requirements in the proposition. To this end, we define:

$$
\left(\begin{array}{rr}
\tilde{\partial}_{3} & \tilde{\partial}_{1} \\
-\tilde{\partial}_{2} & \tilde{\partial}_{4}
\end{array}\right) \equiv l^{-1}\left(\begin{array}{rr}
\partial_{3} & \partial_{1} \\
-\partial_{2} & \partial_{4}
\end{array}\right),
$$

from the construction, $\tilde{\partial}_{3}=\partial / \partial x$ and $-\tilde{\partial}_{2}=\partial / \partial y$. Furtehrmore, since Eqs. (3.7) are also valid for $\tilde{\partial}_{1}, \ldots, \tilde{\partial}_{4}$,

$$
-\tilde{\partial}_{3} u_{n-1}+\tilde{\partial}_{1} u_{n}=0, \quad \tilde{\partial}_{2} u_{n-1}+\tilde{\partial}_{4} u_{n}=0 \quad\left(u_{n}=u_{n}^{1}, u_{n}^{2}\right)
$$

In particular, from Eqs. (3.14) $)_{1}$ it turns out that $\tilde{\partial}_{1} p=1, \tilde{\partial}_{1} q=0, \tilde{\partial}_{4} p=0$ and $\tilde{\partial}_{4} q=1$. This implies that $\tilde{\partial}_{1}=\partial / \partial p+A \partial / \partial x+B \partial / \partial y$ and $\tilde{\partial}_{4}=\partial / \partial q+C \partial / \partial x$ $+D \partial / \partial y$ for some functions $A, B, C$ and $D$. Inserting these formulas into Eqs. $(3.14)_{0}$ one finds:

$$
A=\frac{\partial u_{-1}^{1}}{\partial x}, \quad B=\frac{\partial u_{-1}^{2}}{\partial x}, \quad C=\frac{\partial u_{-1}^{1}}{\partial y}, \quad D=\frac{\partial u_{-1}^{2}}{\partial y}
$$

thus one obtains Eq. (3.11a). Eq. (3.11b) follows from Eqs. (3.14) $)_{-1}$ after simple calculations. This completes the proof of the proposition.

\subsection{Funpther Gemeralizations}

We now turn to the gerenal aspects of the present issue. As we have illustrated for the most simplest case, a number of nonlinear equations that generalize the self-dual Einstein equations from the point of view of integrability can be obtained as the integrability conditions of linear problems of the following type,

$$
D_{\alpha}(\lambda) u=0, \quad \alpha=1, \ldots, r,
$$


with some constraints if necessary, where $D_{\alpha}(\lambda)$ 's are vector fields with prescribed dependence on the parameter $\lambda$ and play the role of unknown functions. The integrability of such a linear problem means, roughly speaking, that it has a maximal number ( $=$ space dimensions $-r$ ) of functionally independent solutions. The integrability conditions in this sense takes the form of commutator relations such as:

$$
\left[D_{\alpha}(\lambda), D_{\beta}(\lambda)\right]=\sum_{\gamma=1}^{r} C_{\alpha \beta}^{\gamma}(\lambda) D_{\gamma}(\lambda),
$$

with appropriate coefficients $C_{\alpha \beta}(\lambda)$ to be determined by $D_{\alpha}(\lambda)$ 's.

For instance, a series of higher dimensional generalizations including the previous example (3.2) can be derived by setting

$$
D_{\alpha}(\lambda)=-\lambda \partial_{\alpha}+\partial_{\alpha+r}, \quad \alpha=1, \ldots, r,
$$

where $\partial_{1}, \ldots, \partial_{2 r}$ are linearly independent vector fields in a $2 r$ dimensional space. The case of $r=2$ agrees with the previous example, and the results obtained therein can be extended to this series of equations just the same way. The results for the present case can be summarized as follows:

i) The linear problem has a set of Laurent series solutions $u^{\alpha}=\sum_{n=-\infty}^{1} u_{n}^{\alpha} \lambda^{n}$ $(\alpha=1, \ldots, r)$ satisfying the condition

$$
d u_{1}^{1} \wedge d u_{1}^{2} \wedge \cdots \wedge d u_{1}^{r} \wedge d u_{0}^{1} \wedge d u_{0}^{2} \wedge \cdots \wedge d u_{0}^{r} \neq 0,
$$

if and only if the following equations are satisfied:

$$
\left[-\lambda \partial_{\alpha}+\partial_{\alpha+r},-\lambda \partial_{\beta}+\partial_{\beta+r}\right]=\sum_{\gamma=1}^{r}\left(C_{\alpha \beta, 0}^{\gamma}+C_{\alpha \beta, 1}^{\gamma}\right)\left(-\lambda \partial_{\gamma}+\partial_{\gamma+r}\right),
$$

where $C_{\alpha \beta, 0}^{\gamma}$ and $C_{\alpha \beta, 1}^{\gamma}$ are some functions independent of $\lambda$ to be determined by the vector fields $\partial_{1}, \ldots, \partial_{2 r}$.

ii) In terms of the 1 -forms $e^{1}, \ldots, e^{2 r}$ defined by the duality relation $\left\langle e^{a}, \partial_{b}\right\rangle=\delta_{b}^{a}$, Eqs. (3.19) are equivalent to the exterior differential equations

$$
d\left(e^{\alpha}+\lambda e^{\alpha+r}\right) \wedge\left(e^{1}+\lambda e^{r+1}\right) \wedge \cdots \wedge\left(e^{r}+\lambda e^{2 r}\right)=0 \quad(\lambda \in \mathbb{C})
$$

iii) The above two representations of the integrability conditions are form-invariant under transformations of the vector fields and 1-forms such as:

$$
\left(\begin{array}{cc}
\partial_{1} & \partial_{r+1} \\
\vdots & \vdots \\
\partial_{r} & \partial_{2 r}
\end{array}\right) \longrightarrow l\left(\begin{array}{cc}
\partial_{1} & \partial_{r+1} \\
\vdots & \vdots \\
\partial_{r} & \partial_{2 r}
\end{array}\right),\left(\begin{array}{cc}
e^{1} & e^{r+1} \\
\vdots & \vdots \\
e^{r} & e^{2 r}
\end{array}\right) \longrightarrow{ }^{t} l^{-1}\left(\begin{array}{cc}
e^{1} & e^{r+1} \\
\vdots & \vdots \\
e^{r} & e^{2 r}
\end{array}\right),
$$

with $l=\left(l_{\alpha}^{\beta}\right)(\alpha, \beta=1, \ldots, r)$ a $\mathrm{GL}(r, C)$-valued function. With an appropriate 
choice of such transformations the vector fields and the 1-forms in question can be set into a canonical form similar to that one shown in Proposition (3.11).

Furthermore, there is a systematic way to generalize the nonlinear constraint employed for the case of the self-dual Einstein equations. For example, for the case of the above series of equations, we take a complex Lie subgroup $G$ of $\mathbb{G L}(r, \mathbb{C})$ and consider

Constraimt (3.22): The $r \times r$ matrix $\left(\partial_{\beta} u^{\alpha}\right)$ takes values in $G$.

This certainly gives a nonlinear constrain as mentioned above; if in particular $r=2$ and $G=\operatorname{SL}(2, C)$, this reproduces the self-dual Einstein equations. A variety of reductions of the "master equations" (3.19) and (3.20) can be derived as such. It should be also remarked that (3.22) is, in fact, equivalent to

Comstraint (3.23): The $r \times r$ matrix $\left(\partial u^{\alpha} / \partial x^{\beta}\right)$ takes values in $G$.

Here we have taken $\left(p^{1}, \ldots, p^{r}, x^{1}, \ldots, x^{r}\right) \equiv\left(u_{1}^{1}, \ldots, u_{1}^{r}, u_{0}^{1}, \ldots, u_{0}^{r}\right)$ as new space coordinates and regarded $u^{\alpha}$ as functions of these coordinates and $\lambda$. The grouptheoretical meaning of the above constraint will be clarified in $\S 4$.

We now conclude this section with a few remarks:

i) It would be worth mentioning that Zakharov and Shabat [7] already argued equations like (3.16). They pointed out that such equations may be interpreted as the condition of the vanishing of "obstruction" for extending the notion of "zero-curvature representations" from ordinary flat spaces to curved spaces. Eq. (3.2), for example, is connected in this sense with the zero-curvature representation (2.14) of the self-dual Yang-Mills equations, and if $\mathbb{E q}$. (3.2) is satisfied (namely "obstruction" vanishes), the curved space version of (2.14) as shown by Zakharov and Shabat enables one to describe self-dual Yang-Mills fields on such a curved space-time after a manner almost parallel to the case of flat space-time. This leads to essentially the same description of self-dual Yang-Mills fields on conformally self-dual space-times as presented by Atiyah, Hitchin and Singer [24], though the latter takes a more geometric formulation and, in addition, conformally self-dual space-times to be characterized by Eq. (3.2) are somewhat special ones. See also the work of Torres del Castillo [25] for a related topic.

ii) Thus it would be quite natural to expect that other interesting examples of Eqs. (3.16) can be likewise obtained in connection with various analogues of the self-dual Yang-Mills equations. In fact, Eqs. (3.19) can be derived in the 
above-mentioned sense from the curved space version of the "A-series" in higher dimensional integrable gauge-field equations introduced and classified by Ward [11]. Furthermore Ward's classification includes, besides the "A-series", three other classes too; the latter also yield examples of Eqs. (3.16) in their curved space version. (The formulation of the examples derived from the "C-series" and "D-type" in Ward's classification requires multidimensional parameters $\left(\lambda_{1}, \ldots, \lambda_{m}\right)$ rather than a single $\left.\lambda_{\text {. }}\right)$

iii) An important question would be whether there are interesting examples of superspace-analogues of Eqs. (3.16). Perhaps some examples will be found in connection with the curved superspace version of the supersymmetric YangMills (constraint) equations; this issue will be discussed elsewhere.

\section{§4. Group-Theoretical Structures in Curved Twistor Construction}

\subsection{Curved Twistor Construction}

The Riemann-Hilbert problem has become a powerful solution technique of nonlinear integrable systems [26]. As Boyer and Plebanski stressed [17, 18], the curved twistor construction has a structure very similar to the RiemannHilbert problem. The analogy, however, seems to be still incomplete; indeed, the Riemann-Hilbert problem can be formulated as a sort of decomposition problem in a group whereas the curved twistor construction, in its original form, does not take such a form. The purpose of this section is to present a reformulation of the curved twistor construction as a decomposition problem in a "group-like" structure, which consequently enables one to compare these two solution techniques on an equal footing. We start with a brief review of the curved twistor construction here.

This construction, due to Penrose [16], produces self-dual Einstein metrics from a class of three dimensional complex manifolds called "curved twistor spaces". Each curved twistor space $\mathscr{T}$ here is endowed with a fibration $\pi: \mathscr{T}$ $\rightarrow \mathbb{P}^{1}$ and a "twisted" 3-form that defines on each fiber of $\pi$ a symplectic structure. The role to space-time is played by the space of holomorphic sections of $\pi$ (i.e. holomorphic maps $s: \mathbb{P}^{1} \rightarrow \mathscr{T}$ with $\pi \circ s=$ identity map) satisfying an additional condition, which ensures that this space has complex dimensions four. Finding the explicit form of this space is the most difficult part of this construction. Because of this difficulty, carrying out the construction to the 
final stage has been done for very limited cases; see [27-30].

To give a more detailed account of the space of holomorphic sections, we here consider the case where $\mathscr{T}$ is covered with just two coordinate patches taking the form of the inverse images $\pi^{-1}(D)$ and $\pi^{-1}(\hat{D})$. Here $D$ and $\hat{D}$ are appropriate discs centered at $\infty$ and 0 , respectively, and covering the Riemann sphere. This is exactly the situation frequently assumed in the literature [16-18, 21, 27-29], and corresponds to considering metrics in a small neighborhood of a space-time point. For such a case one can take coordinates $\left(w^{1}, w^{2}, w^{3}\right)$ on $\pi^{-1}(D)$ and $\left(\hat{w}^{1}, \hat{w}^{2}, \hat{w}^{3}\right)$ on $\pi^{-1}(\hat{D})$ so that they are related on $\pi^{-1}(D \cap \hat{D})$ as:

$$
w^{1}=F^{1}\left(\hat{w}^{1}, \hat{w}^{2}, \hat{w}^{3}\right), w^{2}=F^{2}\left(\hat{w}^{1}, \hat{w}^{2}, \hat{w}^{3}\right), w^{3}=\hat{w}^{3},
$$

where $F^{1}$ and $F^{2}$, usually called "patching functions", are holomorphic on an open set in $\mathbb{C}^{2} \times(D \cap \widehat{D})$ corresponding to $\pi^{-1}(D \cap \hat{D})$ and satisfy:

$$
\frac{\partial\left(F^{1}, F^{2}\right)}{\partial\left(\hat{w}^{1}, \hat{w}^{2}\right)}=\left(\hat{w}^{3}\right)^{-2}
$$

The last condition reflects the presence of the "twisted" 3-form mentioned above.

For our purpose, however, it is more convenient to modify the notion of patching functions as follows. Namely, we write the coordinates on $\pi^{-1}(\hat{D})$ as $(x, y, \lambda)$ rather than $\left(\hat{w}^{1}, \hat{w}^{2}, \hat{w}^{3}\right)$ and "twist" the patiching functions as:

$$
f^{1}(x, y, \lambda)=\lambda F^{1}(x, y, \lambda), \quad f^{2}(x, y, \lambda)=\lambda F^{2}(x, y, \lambda),
$$

calling them also "patching functions". Evidently they satisfy:

$$
\frac{\partial\left(f^{1}, f^{2}\right)}{\partial(x, y)}=1
$$

which means that the patching functions define a one-parameter family (with parameter $\lambda \in D \cap \hat{D})$ of two dimensional canonical transformations $(x, y)$ $\rightarrow\left(f^{1}, f^{2}\right)$ in the $(x, y)$-space, where the $(x, y)$-space is endowed with the same symplectic structure as introduced in $\S 2$. Recognizing patching functions as such is, as we shall see later, the most basic point of view of our group-theoretical interpretation to the curved twistor construction.

If the curved twistor space in question is as such, then, as shown in the references cited above, the problem of describing holomorphic sections of $\pi$ can be reduced to certain functional equations with additional conditions. We formulate the latter problem as:

Problem (4.5). Find four functions $u^{\alpha}=u^{\alpha}(z, \lambda)$ and $\hat{u}^{\alpha}=\hat{u}^{\alpha}(z, \lambda)(\alpha=1,2)$ 
of five variables $(z, \lambda) \equiv\left(z^{1}, \ldots, z^{4}, \lambda\right)$ satisfying the following conditions:

a) $\lambda^{-1} u^{\alpha}$ and $\hat{u}^{\alpha}(\alpha=1,2)$ are holomorphic functions in the domains $S \times D$ and $S \times \hat{D}$, respectively, where $S$ is a domain in the $z$-space.

b) $d u_{1}^{1} \wedge d u_{1}^{2} \wedge d u_{0}^{1} \wedge d u_{0}^{2} \neq 0$, where $d$ denotes the total differentiation with respect to $z$, and $u_{n}^{\alpha}=u_{n}^{\alpha}(z)(n=1,0,-1, \ldots, \alpha=1,2)$ the Laurent coefficients of $u^{\alpha}$, i.e. $u^{\alpha}=\sum_{n=-\infty}^{1} u_{n}^{\alpha} \lambda^{n}$.

c) $u^{\alpha}(z, \lambda)=f^{\alpha}\left(\hat{u}^{1}(z, \lambda), \hat{u}^{2}(z, \lambda), \lambda\right)$ for $(z, \lambda) \in S \times(D \cap \hat{D})$ and $\alpha=1,2$.

Geometrically, this is certainly equivalent to the problem of finding a fourparameter family of holomorphic sections of $\pi$, the parameters $z=\left(z^{1}, \ldots, z^{4}\right)$ then playing the role of space-time coordinates. The second condition (b) is added to avoid singularities in the metric to be constructed. According to Penrose [16], the above problem indeed has a solution as far as the curved twistor space is sufficiently close to the flat twistor space $\mathbb{P}^{3} \backslash \mathbb{P}^{1}$ (for which $f^{1}=x$ and $f^{2}=y$ ); however, finding its explicit form is by no means an easy task, as already mentioned. We therefore assume that we have solved the above problem, and turn to the construction of the metric.

To give an explicit representation of the metric, we now take $\left(u_{1}^{1}, u_{1}^{2}, u_{0}^{1}, u_{0}^{2}\right)$ as new independent variables, and regard $u^{\alpha}$ and $\hat{u}^{\alpha}$ as functions of these variables and $\lambda$; this is indeed possible by virtue of the above condition (b). The construction of the metric then can be achieved as follows.

Proposition (4.6). Given a pair of patching functions satisfying Eq. (4.4), suppose that there are four functions $u^{\alpha}$ and $\hat{u}^{\alpha}(\alpha=1,2)$ that fulfill all the requirements in Problem (4.5); furthermore, take ( $p, q, x, y) \equiv\left(u_{1}^{1}, u_{1}^{2}, u_{0}^{1}, u_{0}^{2}\right)$ as new independent variables in place of $z$. Then for the tetrad

$$
\begin{gathered}
e^{3}=d x-\frac{\partial u_{-1}^{1}}{\partial x} d p-\frac{\partial u_{-1}^{1}}{\partial y} d q, e^{1}=d p, \\
-e^{2}=d y-\frac{\partial u_{-1}^{2}}{\partial x} d p-\frac{\partial u_{-1}^{2}}{\partial y} d q, e^{4}=d q,
\end{gathered}
$$

$u^{\alpha}$ and $\hat{u}^{\alpha}$ satisfy Eqs. (1.8) and (1.9) respectively. In particular, metric (1.1) is self-dual Einstein, and $u^{\alpha}$ and $\hat{u}^{\alpha}$ give its $u$-potentials.

Proof. From (4.4) and (4.5c), $u^{\alpha}$ and $\hat{u}^{\alpha}$ turn out to satisfy:

$$
d u^{1} \wedge d u^{2}=d \hat{u}^{1} \wedge d \hat{u}^{2} .
$$

The right side, if expanded into a Laurent series of $\lambda$, includes only nonnegative powers of $\lambda$. Therefore 


$$
d u^{1} \wedge d u^{2}=\lambda^{2} \omega^{(2)}+\lambda \omega^{(1)}+\omega^{(0)},
$$

where $\omega^{(2)}, \omega^{(1)}$ and $\omega^{(0)}$ denote the same 2-forms as in Eq. (1.10). From the last equation, $\left(\lambda^{2} \omega^{(2)}+\lambda \omega^{(1)}+\omega^{(0)}\right) \wedge\left(\lambda^{2} \omega^{(2)}+\lambda \omega^{(1)}+\omega^{(0)}\right)=0$; this in particular implies that $\omega^{(1)} \wedge \omega^{(0)}=0$ and $\omega^{(0)} \wedge \omega^{(0)}=0$, so one obtains Eqs. (1.13) just as in the proof of Proposition (1.12). With the aid of Eqs. (1.13) one can calculate the right side of Eq. (4.8) as:

$$
\begin{aligned}
& \lambda^{2} \omega^{(2)}+\lambda \omega^{(1)}+\omega^{(0)} \\
& \quad=\left(d x-\frac{\partial u_{-1}^{1}}{\partial x} d p-\frac{\partial u_{-1}^{1}}{\partial y} d q+\lambda d q\right) \wedge\left(d y-\frac{\partial u_{-1}^{2}}{\partial x} d p-\frac{\partial u_{-1}^{2}}{\partial y} d q+\lambda d q\right) .
\end{aligned}
$$

This completes the proof of the proposition.

The curved twistor construction can further be extended to various nonlinear equations as discussed in $\S 3$. We finally illustrate this for the case of Eqs. (3.4). What one has to modify is just to remove the condition that patching functions should satisfy Eq. (4.4).

Proposition (4.10)。 Consider the same situation as assumed in Proposition (4.6), except that the patching functions do not have to satisfy Eq. (4.4). Then for the dual tetrad of the tetrad

$$
\begin{aligned}
e^{3} & =d x-\frac{\partial u_{-1}^{1}}{\partial x} d p-\frac{\partial u_{-1}^{1}}{\partial y} d q, e^{1}=d p, \\
-e^{2} & =d y-\frac{\partial u_{-1}^{2}}{\partial x} d p-\frac{\partial u_{-1}^{2}}{\partial y} d q, e^{4}=d q,
\end{aligned}
$$

$u^{\alpha}$ and $\hat{u}^{\alpha}$ satisfy Eqs. (3.1). In particular, this tetrad gives a solution of Eqs. (3.4).

Proof. It is not hard to derive from (4.5c) the following equation:

$$
\left(\begin{array}{cc}
\frac{\partial u^{1}}{\partial x} & \frac{\partial u^{1}}{\partial y} \\
\frac{\partial u^{2}}{\partial x} & \frac{\partial u^{2}}{\partial y}
\end{array}\right)^{-1}\left(\begin{array}{l}
d u^{1} \\
d u^{2}
\end{array}\right)=\left(\begin{array}{cc}
\frac{\partial \hat{u}^{1}}{\partial x} & \frac{\partial \hat{u}^{1}}{\partial y} \\
\frac{\partial \hat{u}^{2}}{\partial x} & \frac{\partial \hat{u}^{2}}{\partial y}
\end{array}\right)^{-1}\left(\begin{array}{c}
d \hat{u}^{1} \\
d \hat{u}^{2}
\end{array}\right) .
$$

Expanding the both sides into Laurent series of $\lambda$ and equating their coefficients in view of conditions ( $4.5 \mathrm{a})$ and $(4.5 \mathrm{~b})$, one readily finds that

$$
\text { [the both sides of }(4.11)]=\left(\begin{array}{r}
e^{3}+\lambda e^{1} \\
-e^{2}+\lambda e^{4}
\end{array}\right) \text {. }
$$

Now following the argument employed in the proof of Proposition (2.6), one can 
rewrite Eqs. (4.12) into linear differential equations for $u^{\alpha}$ and $\hat{u}^{\alpha}$, which show that $u^{\alpha}$ and $\hat{u}^{\alpha}$ all satisfy Eqs. (3.1). This completes the proof.

\subsection{Pseudo-Groups and Loop Pseudo-Groups}

Suppose that there are given two curved twistor spaces with patching functions $\left(f^{1}, f^{2}\right)$ and $\left(\tilde{f}^{1}, \tilde{f}^{2}\right)$ respectively. Regarding them as two-dimensional transformations in the $(x, y)$-space with a parameter $\lambda$, one may compose them as:

$$
\begin{aligned}
& \left(f^{1}, f^{2}\right) \circ\left(\tilde{f}^{1}, \tilde{f}^{2}\right) \\
& \equiv\left(f^{1}\left(\tilde{f}^{1}(x, y, \lambda), \tilde{f}^{2}(x, y, \lambda), \lambda\right), f^{2}\left(\tilde{f}^{1}(x, y, \lambda), \tilde{f}^{2}(x, y, \lambda), \lambda\right)\right),
\end{aligned}
$$

which gives a new pair of patching functions. This operation is exactly the "nonlinear superposition" of curved twistor spaces due to Boyer and Plebanski [18], who thus introduced to the set of curved twistor spaces of the above type a "group" structure. This however contains a problem. Namely, what really forms a group is, for example, the set of curved twistor spaces with patching functions which are globally defined in $\mathbb{C}^{2} \times(D \cap \widehat{D})$ and give, for every $\lambda$, automorphisms of the whole $(x, y)$-space $\mathbb{C}^{2}$. On the other hand, metrics defined only locally correspond to patching functions defined in smaller domains of $\mathbb{C}^{2}$ $\times(D \cap \hat{D})$, and the composition of such patching functions, (4.13), may become meaningless depending on the configulation of the domains in which they are respectively defined. We here discuss what kind of mathematical structures are relevant to such a situation.

We first consider this issue ignoring the presence of the parameter $\lambda$. Let us call a bijective holomorphic map of an open subset of $\mathbb{C}^{r}$ into another one, for simplicity, a local transformation in $\mathbb{C}^{r}(r \geq 1)$. In terms of the standard coordinates $x=\left(x^{1}, \ldots, x^{r}\right)$ on $\mathbb{C}^{r}$ each local transformation can be represented by an $r$-tuple of functions $f=\left(f^{1}, \ldots, f^{r}\right)$. Just because of the same situation as occuring above, the set of such local transformations does not form a group in a strict sense, though still has a very similar structure. In the mathematical literature such an object is called a "pseudo-group". (For the precise definition of this notion, see $[22,31]$; we omit the detail here). For the description of more special classes of local transformations such as canonical transformations, we take a complex Lie subgroup $G$ of $\mathrm{GL}(r, \mathbb{C})$ and consider the following set of local transformations: 


$$
\begin{aligned}
& \Gamma_{G} \equiv\left\{\text { local transformations } f=\left(f^{1}, \ldots, f^{r}\right) \text {; for each } f\right. \text { the } \\
& \text { Jacobi matrix } \left.\left(\partial f^{\alpha} / \partial x^{\beta}\right) \text { takes values in } G\right\},
\end{aligned}
$$

which evidently is invariant under composition, thus also forms a pseudo-group. For $r=2$ and $\mathrm{G}=\mathrm{SL}(2, \mathbb{C})$ this is nothing but the pseudo-group of local canonical transformations in $\mathbb{C}^{2}$.

We now take into consideration the dependence on the parameter $\lambda$, and regard such local transformations as forming a sort of "loop space". For this purpose, take a circle $C$ in the Riemann sphere with center at 0 , and let $\Gamma_{G}^{C}$ denote the set of analytic maps of $C$ into $\Gamma_{G}$. In other words,

$\Gamma_{G}^{C} \equiv\left\{\right.$ local transformations $f(\lambda)=\left(f^{1}(x, \lambda), \ldots, f^{r}(x, \lambda)\right)$ with a parameter $\lambda$; for each $f(\lambda)$ the parameter $\lambda$ moves on $C$, the coordinate components $f^{\alpha}(x, \lambda)(\alpha=1, \ldots, r)$ depend on $\lambda$ analytically, and for every $\lambda$ the Jacobi matrix $\left(\partial f^{\alpha}(x, \lambda) / \partial x^{\beta}\right)$ takes values in $\left.G\right\}$.

If the circle $C$ is taken so as to be included in the annular domain $D \cap \widehat{D}$, patching functions as used in the aforementioned curved twistor construction can be regarded as elements of $\Gamma_{S L(2, C)}^{C}$ (for the case of the self-dual Einstein equations) or of $\Gamma_{G L(2, \mathbb{C})}^{C}$ (for the case of Eqs. (3.4)). Just as in the case of $\Gamma_{G}$ the composition of elements of $\Gamma_{G}^{C}$, too, may become meaningless; thus $\Gamma_{G}^{C}$ likewise forms a pseudo-group, which we call a loop pseudo-group.

From the point of view of group theory $\Gamma_{G}$ and $\Gamma_{G}^{C}$ both have thus fairly incomplete features. Their infinitesimal counterparts however enjoy more definite realizations as infinite dimensional Lie algebras of vector fields. For $\Gamma_{G}$, the Lie algebra is formed by vector fields $\sum_{\alpha=1}^{r} v^{\alpha}(x) \partial / \partial x^{\alpha}$ for which the Jacobi matrix $\left(\partial v^{\alpha}(x) / \partial x^{\beta}\right)$ take values in the Lie algebra $\mathfrak{g}$ of $G$; let us write this Lie algebra $\gamma_{G}$ for the moment. Then the Lie algebra of $\Gamma_{G}^{C}$ is the loop algebra of $\gamma_{G}$ with respect to $C$ (i.e. the Lie algebra formed by analytic maps of $C$ into $\gamma_{G}$ ), which in particular includes the Lie algebra $\gamma_{G} \otimes \mathbb{C}\left[\lambda, \lambda^{-1}\right]$ of $\gamma_{G}$-valued Laurent polynomials. In the case of $G=\operatorname{SL}(2, \mathbb{C})$ these notions are exactly what Boyer and Plebanski [18] pointed out.

\subsection{Decomposition Problem of Riemanm-Hillbert Type}

Employing the above notions we now show a group-theoretical interpretation of the curved twistor construction. To compare this with the RiemannHilbert problem, let us first briefly explain how the Riemann-Hilbert problem can be formulated in the language of group theory. 
Let $G$ be a complex Lie subgroup of $\mathrm{GL}(r, \mathbb{C})$ and $C$ a circle with center at 0 just as in 4.2. Furthermore, let $G^{C}$ be the set of analytic maps of $C$ into $G$. Each element of $G^{C}$ may thus be regarded as a $G$-valued analytic function $g(\lambda)$ of $\lambda \in C$. By defining the product of two elements as pointwise multiplication of values at each $\lambda \in C, G^{C}$ forms a group which is usually called a loop group. The Riemann-Hilbert problem (to be more precise, regular Riemann-Hilbert problem) then can be formulated as a sort of decomposition problem in a loop group, as follows.

Riemann-Hilbert problem (4.16). For a given element $f(\lambda)$ of $G^{C}$, find two elements $g(\lambda)$ and $h(\lambda)$ of $G^{C}$ that fulfill the following conditions:

a) $g(\lambda)$ and $h(\lambda)$ can be extended, as holomorphic maps into $G$, to some neighborhoods of $C \cup C_{-}$and $C \cup C_{+}$, respectively, where $C_{ \pm}$denote the two connected components of $\mathbb{P}^{1} \backslash C$ with $0 \in C_{+}$and $\infty \in C_{-}$.

b) $g(\infty)=1$.

c) $f(\lambda)=g(\lambda) h(\lambda)^{-1}$ for $\lambda \in C$.

The second condition (b) ensures the uniqueness of $g(\lambda)$ and $h(\lambda)$ if they exist. The above problem can be translated into a linear integral equation of Fredholm type, and a unique solution certainly exists as far as $f(\lambda)$ is sufficiently close to the unit matrix [26].

What we now argue is that the curved twistor construction can be interpreted as a decomposition problem of the following type.

Decomposition problem (4.17). For a given element $f(\lambda)$ of $\Gamma_{G}^{C}$, find two elements $g(\lambda)$ and $h(\lambda)$ of $\Gamma_{G}^{C}$ that fulfill the following conditions:

a) $g(\lambda)$ and $h(\lambda)$ can be extended, as holomorphic maps into $\Gamma_{G}$, to some neighborhoods of $C \cup C_{-}$and $C \cup C_{+}$respectively.

b) $g(\infty)=$ identity transformation.

c) $f(\lambda)=g(\lambda) \circ(\lambda)^{-1}$ for $\lambda \in C$.

Let us rewrite Problem (4.5) into the form of such a decomposition problem. To this end, take the coordinates $(p, q, x, y)$ also here and, regarding $u^{1}, u^{2}, \hat{u}^{1}$ and $\hat{u}^{2}$ as functions of $(p, q, x, y, \lambda)$, define:

$$
\begin{aligned}
& f(\lambda) \equiv\left(f^{1}, f^{2}\right), \\
& g(p, q ; \lambda) \equiv\left(u^{1}-p \lambda, u^{2}-q \lambda\right), \\
& h(p, q ; \lambda) \equiv\left(\hat{u}^{1}, \hat{u}^{2}\right),
\end{aligned}
$$

which then give (as local transformations in the $(x, y)$-space) elements of $\Gamma_{G}^{C}$, 
the latter two elements depending also on the parameters $(p, q)$, where

i) $r=2$ and $G=\operatorname{SL}(2, \mathbb{C})$ for the self-dual Einstein equations,

ii) $r=2$ and $G=G L(2, \mathbb{C})$ for Eqs. (3.4).

Furthermore, by virtue of the condition (a) in (4.5) and of the above choice of coordinates, the conditions (a) and (b) in (4.17) are also fulfilled. Finally, the functional equation (c) in (4.5) can be rewritten as:

$$
T(-p \lambda,-q \lambda) \circ f(\lambda)=g(p, q ; \lambda) \circ h(p, q ; \lambda)^{-1},
$$

where $T(-p \lambda,-q \lambda)$ denotes the translation $(x, y) \rightarrow(x-p \lambda, y-q \lambda)$. Eq. (4.19) evidently takes the form of a decomposition problem as exhibited above, thus we obtain another formulation of the curved twistor construction in the language of loop pseudo-groups. This interpertation can also be extended the same way to the generalised setting discussed in $\S 3$.

Regretfully, the above group-theoretical interpretation yielsd no practical reduction of the difficulty in the original curved twistor construction. Indeed solving the above-mentioned decomposition problem is still a very hard task because of its high nonlinearity. The Riemann-Hilbert problem is, roughly speaking, a linear problem which can be reduced to a linear integral equation of well known Fredholm type. The problem in $\Gamma_{G}^{C}$, on the other hand, is essentially of nonlinear nature; it includes the notion of composition of maps, a typically nonlinear operation. Thus some new technique is required here. This issue is left to researches in the future.

Nevertheless, our group-theoretical point of view seems to indicate various new possibilities of approach to the self-dual Einstein equations and to their generalizations discussed in $\$ 3$. As such an attempt, we shall present in the next section a geometric description of these equations as dynamical motion in an infinite dimensional Grassmann manifold, which gives an extension of the work of Sato [32].

\section{§5. Dymamical Motiom inm Iminimite Dimemsiomal Grassmamm Mamifold}

\subsection{Introduction to This Section}

Sato [32] presented a geometric interpretation of various solution equations as dynamical systems in an infinite dimensional Grassmann manifold. The purpose of this section is to show a similar description of the self-dual Einstein 
equations and their generalizations discussed in $\$ 3$. In order to avoid lengthy and tedeous sequences of inequalities for the check of convergence, we here consider just "formal solutions"; for the case of the self-dual Einstein equations "formal solutions" mean solutions for which $e_{\mu}^{a}(z) \in \mathbb{C}[[z]]$, where $\mathbb{C}[[z]]$ denotes the set of formal power series of space-time coordinates $z=\left(z^{1}, \ldots, z^{4}\right)$ with complex coefficients. (Conditions for their convergence can also be investigated if necessary, though we shall not discuss them here.)

The results presented in this section can be summarized as follows:

i) Formal analogues of loop pseudo-groups $\Gamma_{G}^{C}$, which we call formal loop groups, are introduced. They indeed form groups and, besides, it turns out that the counterpart of decomposition problem (4.17) for these groups always has a unique solution. The notion of formal loop groups thus provides a framework in which the ideas in the preceding section can be realized more rigorously.

ii) Two faithful linear representations of the above groups are introduced. With the aid of one of them the self-dual Einstein equations, as well as their generalizations, can be translated, in their formal solution sector, into some (multi-time) dynamical motion in an infinite dimensional Grassmann manifold.

Arguements developed to establish these resuts are, basically, of the same nature as the cases of solution equations [32] and of integrable gauge-field equations [33-35], though become more complicated because of high nonlinearity underlying the relevant group-theoretical structures (cf. § 4.3). The role to be played by the above-mentioned linear representations is, roughly speaking, to "linearize" that nonlinearity. Indeed the most essential part of the arguments then can be reduced to linear algebra of infinite matrices, thus techniques employed in [33] can be likewise applied to the present case with minimal modifications.

To make the description not too involved, in what follows we deal with just two types of formal loop groups, namely those corresponding to $\Gamma_{\mathrm{GL}(r, C)}^{C}$ and $\Gamma_{\mathrm{SL}(r, C)}^{C}$, and illustrate the above results for these cases alone. Further, in most part of the following arguments, since the complete proof will become too long and mathematically too technical, we indicate just basic ideas of the proof rather than its full detail. 


\subsection{Formal Loop Groups}

Formal analogues of loop groups $G^{c}$ were introduced in [33] also under the name of "formal loop groups". The ideas employed there are also applicable to the present issue to find formal analogues of loop pseudo-groups $\Gamma_{G}^{C}$. We here illustrate this for the cases $G=\mathrm{GL}(r, \mathbb{C})$ and $\mathrm{SL}(r, \mathbb{C})$.

Such a formal analogue should be formed by "formal transformations" which can be represented by $r$-tuples $f=\left(f^{1}, \ldots, f^{r}\right)$ of formal power series of $x=\left(x^{1}, \ldots, x^{r}\right), \lambda$ and $\lambda^{-1}$ of the form

$$
f^{x}=f^{x}(x, \lambda)=\sum_{(I, i) \in Z} f_{I i}^{\alpha} x^{I} \lambda^{-i-1}, Z=\mathbb{N}^{r} \times \mathbb{Z},
$$

where $\mathbb{N} \equiv\{0,1,2, \ldots\}$ (nonnegative integers), $\mathbb{Z} \equiv\{0, \pm 1, \pm 2, \ldots\}$ (ail integers), and $x^{I}$ denotes, for each multi-index $\mathbb{I}=\left(i_{1}, \ldots, i_{r}\right) \in \mathbb{N}^{r}$, the monomial $x^{I}=$

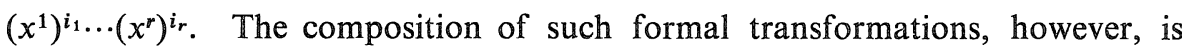
meaningless in general unless some "growth conditions" for the coefficients $f_{I i}^{\alpha}$ are assumed. To give a precise formulation of such "growth conditions" in the present setting, we take as in [33] a $\mathbb{C}$-algebra (an algebra defined over the complex number field) $A$ with the following properties:

Assumption (5.2). $A$ is a commutative $\mathbb{C}$-albegra with a unit element 1 and a filtration $\cdots A_{-2}=A_{-1}=A_{0}=A \supset A_{1} \supset A_{2} \supset \cdots$ formed by complex vector subspaces $A_{n}(n \in \mathbb{Z})$ satisfying the following conditions (a)-(d):

a) $\cap_{n=0}^{\infty} A_{n}=0$.

b) $A_{m} A_{n} \subset A_{m+n}$ for any pair of integers $m$ and $n$.

c) For any sequence $a_{n}(n \geq 0)$ of elements of $A$ with $a_{n} \in A_{n}$, there is a unique element $a$ of $A$ such that $a-\sum_{n=0}^{N} a_{n} \in A_{N+1}$ for every $N \geq 0$. In what follows we write this element $a$ as $\sum_{n=0}^{\infty} a_{n}$; this notation is consistent with the ordinary notion of finite sum.

d) For any integer $m$ and any sequence $a_{n}(n \geq m)$ of elements of $\mathbb{A}$ with $a_{n} \in A_{n}$, the infinite sum $\sum_{n=m}^{\infty} a_{n}$ introduced above belongs to $A_{m}$.

Example (5.3)。

$$
\begin{aligned}
& A=\mathbb{C}[[p, q]]=\left\{\text { formal power series } \sum_{i, j=0}^{\infty} c_{i j} p^{i} q^{j} ; c_{i j} \in \mathbb{C}\right\}, \\
& A_{n}=\left\{\sum_{i, j=0}^{\infty} c_{i j} p^{i} q^{j} ; c_{i j}=0 \text { if } i+j<n\right\} .
\end{aligned}
$$

We now show the definition of formal analogues of $\Gamma_{G}^{C}$ for $G=G \mathbb{L}(r, \mathbb{C})$ and $\operatorname{SL}(r, \mathbb{C})$ : 
Definition (5.4)。

$$
\begin{gathered}
\Gamma_{\mathrm{GL}(r, \mathbb{C})}^{A\langle\lambda\rangle} \equiv\left\{f=\left(f^{1}, \ldots, f^{r}\right) ; f^{\alpha}=\sum_{(I, i) \in Z} f_{I i}^{\alpha} x^{I} \lambda^{-i-1},\right. \\
\left.f_{I i}^{\alpha} \in A_{-|I|-i} \cap A_{-i-1},\left(f_{E_{\beta},-1}^{\alpha}\right) \in \mathrm{GL}(r, \mathbb{C})(\alpha, \beta=1, \ldots, r)\right\}, \\
\Gamma_{\mathrm{SL}(r, \mathbb{C})}^{A\langle\lambda\rangle} \equiv\left\{f=\left(f^{1}, \ldots, f^{r}\right) \in \Gamma_{\mathrm{GL}(r, \mathbb{C})}^{A<\lambda\rangle} ; \partial\left(f^{1}, \ldots, f^{r}\right) / \partial\left(x^{1}, \ldots, x^{r}\right)=1\right\}, \\
\text { where }|I| \equiv i_{1}+\cdots+i_{r} \text { for } I=\left(i_{1}, \ldots, i_{r}\right) \in \mathbb{N}^{r} \text { and } E_{\beta} \equiv(0, \ldots, 1, \ldots, 0) .
\end{gathered}
$$

The coditions imposed on the coefficients $f_{I i}^{\alpha}$ in the above definition are indeed appropriate ones, because under this definition we can obtain the following basic result:

Theorem (5.5)。 $\quad \Gamma_{\mathrm{GL}(r, \mathrm{C})}^{A\langle\lambda\rangle}$ forms a group with respect to the composition $f \circ \tilde{f}$ of two elements $f=\left(f^{1}(x, \lambda), \ldots, f^{r}(x, \lambda)\right)$ and $\tilde{f}=\left(\tilde{f}^{1}(x, \lambda), \ldots, \tilde{f}^{r}(x, \lambda)\right)$ defined as: $f \circ \tilde{f} \equiv\left(f^{1}(\tilde{f}, \lambda), \ldots, f^{r}(\tilde{f}, \lambda)\right)$. Furthermore $\Gamma_{\mathbf{S L}(r, \mathbb{C})}^{A\langle\lambda\rangle}$ forms a subgroup of $\Gamma_{\mathrm{GL}(\boldsymbol{r}, \mathcal{C})}^{A<\lambda\rangle}$.

The proof of the above theorem can be reduced, with the aid of matrix representations as introduced later (see 5.3), to the check of composability and invertibility of corresponding infinite matrices. For the latter problem, techniques as exploited in [33] are likewise applicable with slightest modifications. We omit the detail.

It would be remarkable that unlike loop pseudo-groups $\Gamma_{G}^{C}$, their formal analogues $\Gamma_{G}^{A\langle\lambda\rangle}$ form groups in a strict sense; because of this fact we call them "formal loop groups".

We now show the statement of our result on a formal loop group version of decomposition problem (4.17). To this end, let us introduce the following notion:

Definition (5.6)。

$$
\begin{aligned}
& \Gamma_{G}^{A\langle\lambda\rangle,-} \equiv\left\{f=\left(f^{1}, \ldots, f^{r}\right) \in \Gamma_{G}^{A\langle\lambda\rangle} ; f_{I i}^{\alpha}=0 \text { for } i \leq-2 \text { and } f^{\alpha}(x, \lambda)=x^{\alpha}\right\}, \\
& \Gamma_{G}^{A\langle\lambda\rangle,+} \equiv\left\{f=\left(f^{1}, \ldots, f^{r}\right) \in \Gamma_{G}^{A\langle\lambda\rangle} ; f_{I i}^{\alpha}=0 \text { for } i \geq 0\right\} .
\end{aligned}
$$

Note that $\Gamma_{G}^{A}\langle\lambda\rangle,+$ and $\Gamma_{G}^{A\langle\lambda\rangle,-}$ both form subgroups of $\Gamma_{G}^{A}\langle\lambda\rangle$. The result on the decomposition problem then may be formulated as follows.

Theorem (5.7)。Any element $f$ of $\Gamma_{G}^{A\langle\lambda\rangle}$ can be uniquely decomposed as:

$$
f=g \circ h^{-1}, \quad g \in \Gamma_{G}^{A\langle\lambda\rangle,-}, \quad h \in \Gamma_{G}^{A\langle\lambda\rangle,+} .
$$

The proof of this theorem will be indicated later (see 5.6) in connection with 
the notion of Grassmann manifolds. What seems particularly interesting here is that the counterpart of decomposition problem (4.17) for formal loop groups always has a unique solution. This seems to be a common advantage of the notion of formal loop groups over the analytical ones; indeed it has been shown in [33] that the same situation occurs for the case of formal analogues of loop groups $G^{C}$, too.

\subsection{Limear Representatioms}

We now introduce two linear representations of formal loop groups $\Gamma_{G}^{A<\lambda\rangle}$. The first one, which we write $\rho$, represents each elenent of $\Gamma_{G}^{A\langle\lambda\rangle}$ as an infinite matrix whose rows and columns respectively have $(r+1)$-fold multi-indices:

$$
\rho: \quad \Gamma_{F}^{A\langle\lambda\rangle} \longrightarrow \mathrm{GL}(\mathbb{Z}, A),
$$

where $Z$ is, as already mentioned, the set of multi-indices $\mathbb{N}^{r} \times \mathbb{Z}$, and $\mathbb{G L}(Z, A)$ denotes the set of invertible $Z \times Z$ matrices with matrix elements taken from $A$. The matrix elements of $\rho$ are written

$$
\rho(f)=\left(\rho(f)_{J j}^{I i}\right), \quad f \in \Gamma_{G}^{A\langle\lambda\rangle},
$$

(where the multi-indices $(I, i)$ and $(J, j)$ respectively indicate the rows and columns), and defined as the coefficients of the power series expansion

$$
\sum_{(I, i) \in Z} \rho(f)_{J j}^{I i} x^{J} \lambda^{-j-1}=f^{I} \lambda^{-i-1},
$$

where $f^{I} \equiv\left(f^{1}\right)^{i_{1}} \ldots\left(f^{r}\right)^{i_{r}}$ for $I=\left(i_{1}, \ldots, i_{r}\right) \in \mathbb{N}^{r}$.

Proposition (5.11)。 The map $\rho: \Gamma_{G}^{A<\lambda>\rightarrow G L}(\mathbb{Z}, A)$ thus defined gives a faithful representation of the formal loop group $\Gamma_{G}^{A\langle\lambda\rangle}$.

The second representation, which is to play more important roles later, is the contragredient representation of $\rho$; we write it $\rho^{*}$ for brevity. This also gives a faithful linear representation of $\Gamma_{G}^{A\langle\lambda\rangle}$ :

$$
\rho^{*}: \Gamma_{G}^{A\langle\lambda\rangle} \longrightarrow \mathbb{G L}(\mathbb{Z}, A) .
$$

We write its matrix elements as:

$$
\rho^{*}(f)=\left(\rho^{*}(f)_{I i}^{J j}\right)={ }^{t} \rho(f)^{-1}, \quad f \in \Gamma_{G}^{A\langle\lambda\rangle},
$$

where $(I, i)$ and $(J, j)$ are used, also here, to indicate the rows and columns respectively. It is not hard to see that the matrix elements of $\rho^{*}(f)$ can be reproduced as the coefficients of the power series expansion 


$$
\sum_{(I, i) \in Z} \rho^{*}(f)_{I i}^{J j} x^{I} \lambda^{-i-1}=f^{* J} \lambda^{-j-1},
$$

where we write the coordinate components of the inverse transformation $f^{-1}$ as:

$$
f^{-1}=\left(f^{* 1}, \ldots, f^{* r}\right)
$$

and define: $f^{* J} \equiv\left(f^{* 1}\right)^{j_{1}} \ldots\left(f^{* r}\right)^{j_{r}}$ for $J=\left(j_{1}, \ldots, j_{r}\right) \in \mathbb{N}^{r}$.

Not only being an infinite-matrix representation as above, $\rho^{*}$ turns out to have another more natural realization as a linear representation of $\Gamma_{G}^{A\langle\lambda\rangle}$ in a "formal function space" $A\langle x, \lambda\rangle$ defined as follows:

Definition (5.15).

$$
\begin{aligned}
& A\langle x, \lambda\rangle_{m, n} \equiv\left\{\text { formal series } \xi=\sum_{(I i) \in Z} \xi_{I i} x^{I} \lambda^{-i-1} ; \xi_{I i} \in A_{m-|I|-i} \cap A_{n-i}\right\} \\
& A\langle x, \lambda\rangle_{-\infty, n} \equiv \bigcup_{m \in Z} A\langle x, \lambda\rangle_{m, n}, \\
& A\left\langle x, \lambda \equiv_{m, n \in Z} A\langle x, \lambda\rangle_{m, n} .\right.
\end{aligned}
$$

Note that elements of $A\langle x, \lambda\rangle$ are in one-to-one correspondence with infinite column vectors of size $Z$ as: $\xi \leftrightarrow v(\xi) \equiv\left(\xi_{I i}\right)((I, i) \in Z)$. By using this correspondence another realization of $\rho^{*}$ can be expressed as follows:

Proposition (5.16). For any $f \in \Gamma_{G}^{A\langle\lambda\rangle}$ and any $\xi=\xi(x, \lambda) \in A\langle x, \lambda\rangle$ one has:

$$
v\left(\xi \circ f^{-1}\right)=\rho^{*}(f) v(\xi)
$$

where $\xi \circ f^{-1} \equiv \xi\left(f^{-1}, \lambda\right)$. In other words $\rho^{*}$ can be identified with the action $\xi \rightarrow \xi \circ f^{-1}$ of $\Gamma_{G}^{A\langle\lambda\rangle}$ on $A\langle x, \lambda\rangle$.

We therefore from now on identify the infinite-matrix representation $\rho^{*}$ with the above realization in the $A$-linear space ( $A$-module) $A\langle x, \lambda\rangle$, and use the same sign $\rho^{*}$ to denote the latter one too, i.e.

$$
\rho^{*}(f) \xi=\xi_{\circ} f^{-1}, \quad f \in \Gamma_{G}^{A\langle\lambda\rangle}, \quad \xi \in A\langle x, \lambda\rangle .
$$

The $A$-linear subspaces ( $A$-submodules) $A\langle x, \lambda\rangle_{m, n}(m, n \in \mathbb{Z})$ form a filtration in $A\langle x, \lambda\rangle$. One may consider this filtration as defining a scale for measuring, for example, the convergence of series or sequences in $A\langle x, \lambda\rangle$, just the same way as in the case of the filtration $A_{n}(n \in \mathbb{Z})$ in $A$. In other words the filtration defines, as a system of fundamental neighborhoods at the origin, the structure of a topological $A$-linear space in $A\langle x, \lambda\rangle$. Since

$$
\rho^{*}(f) A\langle x, \lambda\rangle_{m, n}=A\langle x, \lambda\rangle_{m, n} \quad(m, n \in \mathbb{Z})
$$


for every $f \in \Gamma_{G}^{A\langle\lambda\rangle}, \rho^{*}(f)$ becomes a continuous $A$-linear automorphism of $A\langle x, \lambda\rangle$ with respect to this topological structure.

\subsection{Grassmanna Mamifolds}

In the mathematical literature Grassmann manifolds in question are usually finite dimensional; a typical example is the Grassmann manifold formed by $n$-dimensional vector subspaces of a fixed $N$-dimensional vector space $(n \leq N)$. Infinite dimensional ones, however, are also investigated to some extent. Indeed Sato [32] introduced an infinite dimensional Grassmann manifold $\mathrm{GM}=\mathrm{GM}_{V}$ starting from an infinite dimensional complex vector space $V$. Here $V$ is endowed with a filtration $\cdots \supset V_{-1} \supset V_{0} \supset V_{1} \supset \cdots$ formed by vector subspaces $V_{n} \subset V(n \in \mathbb{Z})$, which defines the structure of a topological vector space in $V$ by taking $V_{n}$ 's as a system of fundamental neighborhoods at the origin. $\mathbb{G M}_{V}$ is then defined as:

(5.19) $\mathrm{GM}_{V} \equiv$ closed vector subspaces $U \subset V$; for each $U$ the dimensions of the kernel and co-kernel of the natural linear map $U \rightarrow V / V_{0}$ (induced by the projection of $V$ onto the quotient vector space $\left.V / V_{0}\right)$ are both finite and coincide .

We now apply the same construction to the following complex vector space $\mathbb{C}\langle x, \lambda\rangle$.

\section{Definition (5.20)。}

$$
\begin{aligned}
& \mathbb{C}\langle x, y\rangle_{m, n} \equiv\left\{\text { formal series } \xi=\sum_{(I, i) \in Z} \xi_{I i} x^{I} \lambda^{-i-1} ; \xi_{I i} \in \mathbb{C} \text { for }(I, i) \in \mathbb{Z},\right. \\
&\text { and } \left.\xi_{I i}=0 \text { if }|I|+i<m \text { or } i<n\right\}, \\
& \mathbb{C}\langle x, \lambda\rangle_{-\infty, n} \equiv \bigcup_{m \in \mathbb{Z}} \mathbb{C}\langle x, \lambda\rangle_{m, n}, \\
& \mathbb{C}\langle x, \lambda\rangle \equiv \bigcup_{m, n \in Z} \mathbb{C}\langle x, \lambda\rangle_{m, n} .
\end{aligned}
$$

This is evidently a special case of $A\langle x, \lambda\rangle$ in which the basic algebra $A$ is set equal to the complex number field $\mathbb{C}$ endowed with the trivial filtration: $\mathbb{C}_{n}=\mathbb{C}$ for $n \leq 0$ and $\{0\}$ for $n>0 . \quad \mathbb{C}\langle x, \lambda\rangle$ becomes a topological vector space with $\mathbb{C}\langle x, \lambda\rangle_{m, n}(m, n \in \mathbb{Z})$ a system of fundamental neighborhoods at the origin. Furthermore the subspaces $\mathbb{C}\langle x, \lambda\rangle_{-\infty, n}(n \in \mathbb{Z})$ give a filtration similar to $V_{n}(n \in \mathbb{Z})$ in $V$. It would be therefore reasonable to introduce the following analogue of Sato's Grassmann manifold $\mathrm{GNi}_{V}$. 
Defimition (5.21)。

$\mathrm{GM}_{\boldsymbol{C}\langle x, \lambda\rangle} \equiv\{$ closed vector subspaces $U \subset \mathbb{C}\langle x, \lambda\rangle$; for each $U$ the dimensions of the kernel and co-kernel of the natural linear map $U \rightarrow \mathbb{C}\langle x, \lambda\rangle \mid \mathbb{C}\langle x, \lambda\rangle_{-\infty, 0}$ are both finite and coincide .

Remark. At present, it is not altogether confirmed whether this is really an appropriate definition of a Grassmann manifold to be constructed from the vector space $\mathbb{C}\langle x, \lambda\rangle$, because the structure of this vector space is far more complicated than that of $V$. We shall not go further into discussions on this issue, and take the above definition as a "working hypothesis".

Actually, what is relevant to the description of formal solutions of the nonlinear equations argued later is a subset of $\mathrm{GM}_{\mathbb{C}\langle x, \lambda\rangle}$ defined below, rather than the whole $\mathrm{GM}_{\boldsymbol{C}\langle x, \lambda\rangle}$.

Definition (5.22)。

$\mathrm{GM}_{\mathbb{C}\langle x, \lambda\rangle}^{\phi} \equiv\{$ closed linear subspaces $U \subset \mathbb{C}\langle x, \lambda\rangle$; for each $U$ the natural linear map $U \rightarrow \mathbb{C}\langle x, \lambda\rangle / \mathbb{C}\langle x, \lambda\rangle_{-\infty, 0}$ is an isomorphism $\}$.

This subset is introduced as an analogue of the Schubert cell $\mathrm{GM}_{V}^{\emptyset}$ of $\mathrm{GM}_{V}$ [32] (where $\varnothing$ denote the empty Young tableau) which for a finite dimensional case corresponds to the open dense cell. A specific feature of this subset is that for each $U \in \mathrm{GM}_{C<x, \lambda>}^{\emptyset}$ there is a basis $\left\{\xi^{J j} ;(J, j) \in N^{c}\right\} \quad\left(N^{c} \equiv \mathbb{N}^{r} \times \mathbb{N}^{c}, \mathbb{N}^{c}\right.$ $\equiv \mathbb{Z} \backslash \mathbb{N})$ of $U$ as a vector space which satisfies the following condition:

$$
\xi^{J j}-x^{J} \lambda^{-j-1} \in \mathbb{C}\langle x, \lambda\rangle_{-\infty, 0} \quad \text { for }(J, j) \in N^{c} .
$$

Such a basis is unique, and can be obtained as the inverse images of $\left[x^{J} \lambda^{-j-1}\right]$, $(J, j) \in N^{c}$, by the linear isomorphism $U \rightarrow \mathbb{C}\langle x, \lambda\rangle / \mathbb{C}\langle x, \lambda\rangle_{-\infty, 0}$. Here the sign $\left[x^{J} \lambda^{-j-1}\right]$ denotes the image of $x^{J} \lambda^{-j-1}$ by the projection of $\mathbb{C}\langle x, \lambda\rangle$ onto $\mathbb{C}\langle x, \lambda\rangle / \mathbb{C}\langle x, \lambda\rangle_{-\infty, 0}$.

\subsection{Variants}

In actual applications (see 5.6 and 5.7) we need the following variants of $\mathrm{GM}_{\boldsymbol{C}\langle x, \lambda\rangle}^{\emptyset}$ :

Definition $(5.24)$.

$\mathrm{GM}_{A \otimes C}^{\emptyset}\langle x, \lambda\rangle \equiv$ closed $A$-linear subspaces $\left.U \subset A \otimes C<x, \lambda\right\rangle$; for each $U$ the natural $A$-linear map $U \rightarrow A \otimes C\langle x, \lambda\rangle \mid A \otimes C\langle x, \lambda\rangle_{-\infty, 0}$ is an isomorphism $\}$, where the tensor product $A \otimes \mathbb{C}\langle x, \lambda\rangle=A \otimes_{\mathbb{C}} \mathbb{C}\langle x, \lambda\rangle$ is endowed with the structure of a topological $A$-linear space in which a system of fundamental 
neighborhoods at the origin is given by $A \otimes \mathbb{C}\langle x, \lambda\rangle_{m, n}(m, n \in \mathbb{Z})$.

Defimition (5.25)。

$\mathrm{GM}_{A\langle x, \lambda\rangle}^{\emptyset} \equiv\{$ closed $A$-linear subspaces $U \subset A\langle x, \lambda\rangle$; for each $U$ the natural $A$-linear map $U \rightarrow A\langle x, \lambda\rangle \mid A\langle x, \lambda\rangle_{-\infty, 0}$ is an isomorphism $\}$.

Roughly speaking, the first variant $\mathrm{GM}_{A \otimes C<x, \lambda>}^{\varnothing}$ represents a sort of "map space" with values in $\mathrm{GM}_{\mathbb{C}\langle x, \lambda\rangle}^{\emptyset}$. For example, if $A=\mathbb{C}[[p, q]]$ (see Example (5.3)), each element of this space may be interpreted as a "formal map" of the $(p, q)$-space into $\mathrm{GM}_{\mathbb{C}\langle x, \lambda\rangle}^{\emptyset}$. Evidently one may take this geometric picture as a formulation of (multi-time) dynamical motion in $\mathbb{G M}_{\mathbb{C}\langle x, \lambda>}^{\mathscr{Q}}$.

The interpretation of the second variant $\mathrm{GM}_{A<x, \lambda\rangle}^{\Phi}$, on the other hand, is seemingly less clear from the definition. There is however a direct connection with the first variant as follows, and from this fact we can see that these two variants represent essentially the same object.

Proposition (5.26). Regard $A \otimes \mathbb{C}\langle x, \lambda\rangle$ as an A-linear subspace of $A\langle x, \lambda\rangle$ after a natural manner, and define for each $U \in \mathbb{G M}_{A<x, \lambda\rangle}^{\Phi}$ an $A$ linear subspace $\iota(U)$ of $A \otimes \mathbb{C}\langle x, \lambda\rangle$ as: $\iota(U) \equiv \mathbb{U} \cap A \otimes \mathbb{C}\langle x, \lambda\rangle$. Then $\iota(U)$

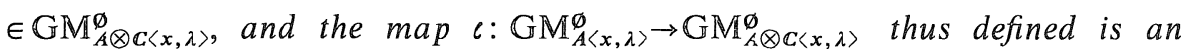
bijection.

Sketch of proof. The most crucial part of the proof is to show that $U$ and $\iota(U)$ have a common basis $\left\{\xi^{J j} ;(J, j) \in N^{c}\right\}$ such that

$$
\xi^{J j}-x^{J} \lambda^{-j-1} \in A \otimes \mathbb{C}\langle x, \lambda\rangle_{-\infty, 0} \quad \text { for } \quad(J, j) \in N^{c} .
$$

Once the existence of such a basis is ensured, it is not very hard to check the statements in the proposition by employing that basis. So let us consider how to obtain such a basis. Since the natural $A$-linear map $U \rightarrow A\langle x, \lambda\rangle \mid A\langle x, \lambda\rangle_{-\infty, 0}$ is an isomorphism, the inverse images of $\left[x^{J} \lambda^{-j-1}\right],(J, j) \in N^{c}$, where the sign $\left[x^{J} \lambda^{-j-1}\right]$ denotes the image of $x^{J} \lambda^{-j-1}$ by the projection of $A\langle x, \lambda\rangle$ onto $A\langle x, \lambda\rangle \mid A\langle x, \lambda\rangle_{-\infty, 0}$, form a basis $\left\{\tilde{\xi}^{J j} ;(J, j) \in N^{c}\right\}$ of $U$ such that

$$
\tilde{\xi}^{J j}-x^{J} \lambda^{-j-1} \in A\langle x, \lambda\rangle_{-\infty, 0} \quad \text { for }(J, j) \in N^{c} .
$$

We now examine the $Z \times N^{c}$ matrix $\tilde{M} \equiv\left(\tilde{\xi}_{I i}^{J j}\right)\left((I, i) \in \mathbb{Z},(J, j) \in N^{c}\right)$ formed by the column vectors $v\left(\tilde{\xi}^{J j}\right)=\left(\tilde{\xi}_{I i}^{J j}\right)$. Applying techniques developed in [33] with appropriate modifications, one can show that the $N^{c} \times N^{c}$ part $\tilde{M}_{(-)}$of $\tilde{M}$ is an invertible matrix and that the product $\tilde{M} \tilde{M}_{(-)}^{-1}$ also makes sense. We then write the matrix elements of this product matrix as: 


$$
\tilde{M} \tilde{M}_{(-)}^{-1}=\left(\xi_{I i}^{J j}\right) \quad\left((I, i) \in Z,(J, j) \in N^{c}\right),
$$

and define: $\xi^{J j} \equiv \sum \xi_{I i}^{J j} x^{I} \lambda^{-j-1}$ for $(J, j) \in N^{c}$. This yields a basis we have sought for.

Remark. The fact that $U$ and $c(U)$ have a common basis does not means that they coincide. This is because the topological structure of $A \otimes C\langle x, \lambda\rangle$ (see Definition (5.24)) is distinct from the one to be induced from that of $A\langle x, \lambda\rangle$. Indeed $\iota(U)$ is in general not a closed subspace of $A\langle x, \lambda\rangle$, but its closure in $A\langle x, \lambda\rangle$ agrees with $U$. This, in particular, explains how the inverse of the map $\iota$ can be constructed.

\subsection{Link between Formal Loop Groups and Grassmann Manifolds}

The following theorem is the key to connect the notion of formal loop groups with a Grassmann manifold.

Theorem (5.30). For every element $f$ of $\Gamma_{G}^{A\langle\lambda\rangle}$ the A-linear subspace $\gamma(f) \equiv \rho^{*}(f) A\langle x, \lambda\rangle_{(-)}$of $A\langle x, \lambda\rangle$, where $A\langle x, \lambda\rangle_{(-)} \equiv\left\{\xi=\sum \xi_{I i} x^{I} \lambda^{-i-1} \in\right.$ $A\langle x, \lambda\rangle ; \xi_{I i}=0$ for $\left.i \geq 0\right\}$, becomes an element of $\mathrm{GM}_{A\langle x, \lambda\rangle}^{\varnothing}$.

Sketch of proof. Since $\rho^{*}(f)$ is a continuous $A$-linear automorphism of $A\langle x, \lambda\rangle$ (see 5.3), the check of the closedness of $\gamma(f)$ can be reduced to that of $A\langle x, \lambda\rangle_{(-)}$, but the latter is not a hard task; we omit the detail. On the other hand, in order to show that the natural $A$-linear map of $\gamma(f)$ into $A\langle x, \lambda\rangle \mid$ $A\langle x, \lambda\rangle_{-\infty, 0}$ is an isomorphism, it is sufficient to find a basis of $\gamma(f)$ satisfying condition (5.27) or (5.28). We can indeed construct such a basis, using basically the same ideas as employed in the proof of Proposition (5.26), as follows. First define: $\tilde{\xi}^{J j} \equiv \rho^{*}(f) x^{J} \lambda^{-j-1}$ for $(J, j) \in N^{c}$. They evidently form a basis of $\gamma(f)$, which however does not satisfy condition (5.27) nor (5.28). To find an appropriate basis, consider the $Z \times N^{c}$ matrix $\tilde{\tilde{M}} \equiv\left(\tilde{\tilde{\xi}}_{I i}^{j}\right)\left((J, j) \in Z,(I, i) \in N^{c}\right)$ made up of the column vectors $v\left(\tilde{\tilde{\xi}}^{J j}\right)=\left(\tilde{\tilde{\xi}}_{I i}^{j}\right)$. Let $\tilde{\tilde{M}}_{(-)}$denote its $N^{c} \times N^{c}$ part; then just as in the case of the matrix $\tilde{M}$ (see the proof of Proposition (5.26)) the product matrix $\tilde{\tilde{M}} \tilde{\tilde{M}}_{(-)}^{-1}$ turns out to make sense, and from its column vectors we obtain a basis $\left\{\xi^{J j} ;(J, j) \in N^{c}\right\}$ of $\gamma(f)$ that satisfies condition (5.27). The theorem can be proved along this line.

With the aid of the basis $\left\{\xi^{J j} ;(J, j) \in N^{c}\right\}$ constructed above, besides, we can prove Theorem (5.7). Let us first consider the case of $G=G L(r, \mathbb{C})$. From the above basis, pick out the elements corresponding to $(J, j)=\left(E_{\alpha},-1\right)$ 
(cf. Definition (5.4)) and write them as: $g^{* \alpha}, \alpha=1, \ldots, r$. Evidently, $g^{*} \equiv$

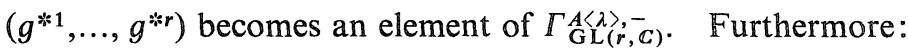

Proposition (5.31)。 The inverse of $g^{*}, g \equiv\left(g^{*}\right)^{-1}$, gives the first factor of the decomposition in the statement of Theorem (5.7).

Proof. Since $g^{* \alpha} \in \gamma(f)=\rho^{*}(f) A\langle x, \lambda\rangle_{(-)}, g^{* \alpha} \circ f(\alpha=1, \ldots, r)$ are elements of $A\langle x, \lambda\rangle_{(-)}$. This implies that the composition $h \equiv f^{-1} \circ g=\left(g^{-1} \circ f\right)^{-1}$ is an element of $\left.\Gamma_{\mathrm{GL}}^{A<\langle<,+}, \boldsymbol{C}\right)$, thus gives the second factor of the decomposition. This completes the proof of the proposition.

For the case of $G=\mathrm{SL}(r, \mathbb{C})$ we first decompose the given element $f$ of $\Gamma_{\mathrm{SL}(\boldsymbol{r}, \mathbb{C})}^{A\langle\lambda\rangle}$ as an element of $\Gamma_{\mathrm{GL}(r, \boldsymbol{C})}^{A\langle\lambda\rangle}$ as: $f=g \circ h^{-1}$ for some $g \in \Gamma_{\mathrm{GL}(r, \mathbb{C})}^{A\langle\lambda\rangle,-}$ and $h \in$ $\Gamma_{\mathrm{GL}(r, \boldsymbol{C})}^{A\langle\lambda\rangle,+}$. In fact, it then turns out that $g$ and $h$ become elements of $\Gamma_{\mathrm{SL}(r, \bar{C})}^{A\langle\lambda\rangle,}$ and $\Gamma_{\mathrm{SL}}^{A\langle\lambda\rangle, \mathbb{C})}$, respectively. To see this, we use the following equation for their Jacobi determinants:

$$
\partial(g) / \partial(x)=\partial(f) /\left.\partial(x)\right|_{x \rightarrow h} \partial(h) / \partial(x)=\partial(h) / \partial(x) .
$$

Let us note here that the right side includes just nonnegative powers of $\lambda$, whereas the left side takes a form such as: $1+$ (linear combination of $\left.\lambda, \lambda^{2}, \lambda^{3}, \ldots\right)$. Thus $\partial(g) / \partial(x)=\partial(h) / \partial(x)=1$.

Remarks. i) The map $\gamma: \Gamma_{G}^{A}\langle\lambda\rangle \rightarrow \mathrm{GM}_{A\langle x, \lambda\rangle}^{\emptyset}$ defined above is invariant under the right-multiplication of elements of $\Gamma_{G}^{A\langle\lambda\rangle,+}$ (i.e. $\gamma(f \circ h)=\gamma(f)$ for every $\left.h \in \Gamma_{G}^{A}\langle\lambda\rangle,+\right)$ and the induced map $[\gamma]: \Gamma_{G}^{A\langle\lambda\rangle} \mid \Gamma_{G}^{A\langle\lambda\rangle,+} \rightarrow \mathbb{G M}_{A\langle x, \lambda\rangle}^{\emptyset}$ is an injection. Thus we obtain an embedding of the quotient space $\Gamma_{G}^{A\langle\lambda\rangle} \mid \Gamma_{G}^{A\langle\lambda\rangle,+}$ into $\mathrm{GM}_{A<x, \lambda>}^{\emptyset}$.

ii) Theorem (5.7) shows that the natural map $\Gamma_{G}^{A\langle\lambda\rangle,-} \rightarrow \Gamma_{G}^{A\langle\lambda\rangle} \mid \Gamma_{G}^{A\langle\lambda\rangle,+}$ induced by the projection of $\Gamma_{G}^{A\langle\lambda\rangle}$ onto $\Gamma_{G}^{A\langle\lambda\rangle} \mid \Gamma_{G}^{A}\langle\lambda\rangle,+$ is an bijection. Its composition with the map $[\gamma]$ agrees with the restriction of the map $\gamma$ onto $\Gamma_{G}^{A}\langle\lambda\rangle,-$.

iii) The map $\gamma$, or equivalently the map $[\gamma]$, is not a surjection. It is not hard to specify the structure of the image, though we omit the detail.

\subsection{Description of Formal Solutions}

We now turn to the description of formal solutions of Eqs. (1.4) and (3.4). It is quite straight forward to rewrite the setting in $\S 4$ into its formal counterpart for formal loop groups. The relevant formal loop groups are:

i) $\Gamma_{\mathrm{SL}(2, \mathcal{C})}^{\mathcal{C}}[p]\langle i\rangle$ for the case of Eqs. (1.4), 
ii) $\Gamma_{G L(2, \mathcal{C})}^{C[p, q]}\langle\lambda\rangle$ for the case of Eqs. (3.4), where the $\mathbb{C}$-algebra of formal power series $\mathbb{C}[[p, q]]$ is endowed with the filtration exihbited in Example (5.3). The counterpart of the data $f(\lambda)$ representing a pair of patching functions is now taken from:

iii) $\Gamma_{\mathrm{SL}(2, \boldsymbol{C})}^{\mathbb{C}\langle\lambda\rangle}$ for the case of Eqs. (1.4),

iv) $\Gamma_{\mathrm{GL}(2, C)}^{C<\lambda>}$ for the case of Eqs. (3.4),

where $\Gamma_{G}^{\mathbb{C}\langle\lambda\rangle}(G=\mathrm{GL}(2, \mathbb{C}), \operatorname{SL}(2, \mathbb{C}))$ denotes the special case of $\Gamma_{G}^{A\langle\lambda\rangle}$ in which $A$ is set equal to the complex number field $\mathbb{C}$ endowed with the trivial filtration: $\mathbb{C}_{n}=\mathbb{C}$ for $n \leq 0$ and $\{0\}$ for $n>0$. If such an element $f(\lambda)$ is given, the decomposition problem relevant to these equations can be written:

$$
\begin{aligned}
& T(-p \lambda,-q \lambda) \circ f(\lambda)=g(p, q ; \lambda) \circ h(p, q ; \lambda)^{-1}, \\
& g(p, q ; \lambda) \in \Gamma_{G}^{\mathbb{G}}[[p, q]]\langle\lambda\rangle,-, \quad h(p, q ; \lambda) \in \Gamma_{G}^{\mathbb{G}}[[p, q]]\langle\lambda\rangle,+.
\end{aligned}
$$

By virtue of Theorem (5.7) such factors $g(p, q ; \lambda)$ and $h(p, q ; \lambda)$ do exist for every $f(\lambda)$. From the coordinate components of $g(p, q ; \lambda)$ and $h(p, q ; \lambda)$ we define $u^{1}, u^{2}, \hat{u}^{1}$ and $\hat{u}^{2}$ as in (4.18). Then tracing back the arguements in $\S 4$, we readily find that the statements of Propositions (4.6) and (4.10) are also valid in the present case.

The above solution process can be reinterpreted from a somewhat different point of view. Let the sign $[f]$ denote, for each element $f$ of $\Gamma_{G}^{A\langle\lambda\rangle}$, the image of $f$ by the projection of $\Gamma_{G}^{A\langle\lambda\rangle}$ onto $\Gamma_{G}^{A\langle\lambda\rangle} \mid \Gamma_{G}^{A}\langle\lambda\rangle,+$. It then turns out that the "dynamics" of $[g(p, q ; \lambda)] \in \Gamma_{G}^{\mathbb{C}[[p, q]]\langle\lambda\rangle} / \Gamma_{G}^{\mathbb{G}}[[p, q]\}\langle\lambda\rangle,+$ is governed by a very simple law. To see thi i, note first that from (5.33), by letting $p, q \rightarrow 0$,

$$
f(\lambda)=g(0,0 ; \lambda) \circ h(0,0 ; \lambda)^{-1} .
$$

Inserting this relation into (5.33) and taking the projection onto the quotient space of the formal loop groups, we obtain:

$$
[T(-p \lambda,-q \lambda) \circ g(0,0 ; \lambda)]=[g(p, q ; \lambda)] .
$$

Thus the "time-evolution" $[g(0,0 ; \lambda)] \rightarrow[g(p, q ; \lambda)]$, where $p$ and $q$ are regarded as multi-time parameters, is realized here as the action (by left-multiplication) of $T(-p \lambda,-q \lambda)$ on $\Gamma_{G}^{\mathbb{C}}[[p, q]]\langle\lambda\rangle \mid \Gamma_{G}^{\mathbb{C}}[[p, q]]\langle\lambda\rangle,+$. The above formula is of course valid for any "initial data" $g(0,0 ; \lambda) \in \Gamma_{G}^{C}\langle\lambda\rangle$ and, in fact, it is not hard to see that any formal solution of Eqs. (1.4) or (3.4) can be produced by this process.

The above group-theoretical picture can be transferred, by the map $\gamma$ or $\gamma^{\prime}$ $\equiv \iota \circ \gamma$, to (multi-time) dynamical motion in the Grassmann manifold $\mathrm{GM}_{\mathbb{C}\langle x, y}^{\emptyset}$ 
$(r=2)$, where we write the variables $\left(x_{1}, x_{2}\right)$ as $(x, y)$ after the notation in the preceding sections. To be more precise, we consider the dynamics of the "moving point" $\gamma(g(p, q ; \lambda))$ or $\gamma^{\prime}(g(p, q ; \lambda))$. The dynamics of the former takes a particularly simple form as follows.

Theorem (5.36). The "time-evolution" $\quad \gamma(g(0,0 ; \lambda)) \rightarrow \gamma(g(p, q ; \lambda)) \quad$ is generated by the action of the linear differential operator $\exp (p \lambda \partial / \partial x+q \lambda \partial / \partial y)$ $\equiv \sum_{k=0}^{\infty}(p \lambda \partial / \partial x+q \lambda \partial / \partial y)^{k} / k !$ as:

$$
\gamma(g(p, q ; \lambda))=\exp (p \lambda \partial / \partial x+q \lambda \partial / \lambda y) \gamma(g(0,0 ; \lambda)) .
$$

Proof. From (5.35) and from the definition of the map $\gamma$

$$
\gamma(g(p, q ; \lambda))=\rho^{*}(T(-p \lambda,-q \lambda)) \gamma(g(0,0 ; \lambda)) .
$$

On the other hand it is not hard to check that

$$
\rho^{*}(T(-p \lambda,-q \lambda))=\exp (p \lambda \partial / \partial x+q \lambda \partial / \partial y) .
$$

This completes the proof.

It should be noted here that not just on the image of $\gamma$, the differential operator $\exp (p \lambda \partial / \partial x+q \lambda \partial / \partial y)$ induces also a map of $\mathbb{G M}_{\mathscr{C}\langle x, y, \lambda\rangle}^{\Phi}$ into $\left.\operatorname{GM}_{C}^{\alpha}[[p, q]\} x, y, \lambda\right\rangle$. (This can be proved just the way as the proof of Proposition (5.26) and Theorem (5.30), using appropriate bases as employed therein.) According to what we observed in 5.5, this map represents, in an abstract sense, some dynamical motion in GMi $\underset{C}{\varnothing} x, y, \lambda>$ with "formal" multi-time parameters $(p, q)$. The image of $\gamma$ forms an "invariant subset" of this dynamical motion, and on this subset, as the above theorem shows, the induced dynamical motion exactly describes how the point $\gamma(g(p, q ; \lambda))$ "moves" depending on the multitime parameters $(p, q)$. This conclusion is quite parallel to the cases of soliton equations discussed by Sato [32] and of gauge-field equations by Suzuki, Harnad, Jacques, and the author [33-35]. It is also possible, as in [33-35], to reformulate the present setting in terms of some infinite matrices and differential equations to describe the dynamics of these infinite matrices. The matrices $\tilde{M}$, $\tilde{\tilde{M}}$, etc. (see 5.5 and 5.6 ), can be used for such a formulation.

\section{Comelusion}

We have investigated the self-dual Einstein equations and related equations from the point of view of integrability. In particular the following three aspects 
have been discussed in detail: i) The existence of a linear scattering problem. ii) A group-theoretical description of solutions with the aid of a decomposition problem similar to the Riemann-Hilbert problem. iii) A geometric interpretation as dynamical motion in an infinite dimensional Grassmann manifold. It would be remarkable that the group-thoretical structures relevant to this case have turned out to be somewhat different from those of classical examples of nonlinear integrable systems. This implies that the self-dual Einstein equations may be thought of as an essentially new type of nonlinear integrable system. We have also shown a general framework to produce this type of nonlinear integrable systems, which seems to include a broad class of new examples of such nonlinear systems.

Some problems however remain open. For example, the process of how to find the exact form of special solutions (like solitons for various solution equations, instantons and monopoles for the self-dual Yang-Mills equations, etc.) is still not very clear in our description of solutions. This issue is left to reseaches in the future. Another issue to be explored is to make clear the relation of our method with other ones that have not been mentioned in the text. In this respect Sanchéz's work [36] also dealing with some aspects of integrability and Gindikin's work [37] borrowing ideas from integral geometry, will provide particularly interesting material. Some new developments may be expected in these directions.

\section{Acknowledgement}

The author is deeply grateful to Prof essor M. Sato and Dr. M. Noumi for much valuable advice.

\section{References}

[1] "Bäcklund transformations, the Inverse Scattering Method, Soliton and Their Applications", ed. R. M. Miura, Lect. Notes. Math., 515, Springer-Verlag 1976.

[2] "Group Theoretical Methods in Physics", ed. M. Serdaroglu and E. Inönü, Lect. Notes. Phys., no. 180, Springer-Verlag 1983.

[ 3 ] “Nonlinear Phenomena", ed. K. B. Wolf, Lect. Notes. Phys., 189, Springer-Verlag 1983.

[4] Jimbo, M., and Miwa, T., Solitons and infinite dimensional Lie algebras, Publ. RIMS, 19 (1983), 943-1001.

[5] "Integrable Quantum Field Theories", ed. H. Hietarinta and C. Montonen, Lect. Notes. Phys., 151, Springer-Verlag 1982. 
[6] Belavin, A. A. and Zakharov, V. E., Yang-Mills equations as inverse scattering problem, Phys. Lett., 73B (1978), 53-57.

[7] Zakharov, V. E. and Shabat, A. A., Integration of nonlinear equations of mathematical physics by the method of inverse scattering II, Func. Anal. Appl., 13 (1979), 166-174.

[8] Pohlmeyer, K., On the Lagrangian theory of anti-self-dual fields in four dimensional Euclidean space, Commun. Math. Phys., 72 (1980), 37-47.

[9] Chau, L.-L., Prasad, M. K., and Sinha, S., Some aspects of the linear systems for self-dual Yang-Mills fields, Phys. Rev., D24 (1981), 1574-1580.

[10] Ueno, K. and Nakamura, Y., Transformation theory for anti-self-dual equations and the Riemann-Hilbert problem, Phys. Lett., $109 \mathbb{B}$ (1982), 273-278.

- Transformation theory for anti-self-dual equations, Publ. RIMS, 19 (1983), 519-547.

[11] Ward, R. S., Completely solvable gauge-field equations in dimensions greater than four, Nucl. Phys., B236 (1984), 381-396.

[12] Volovich, I. V., Supersymmetric Yang-Mills equations as an inverse Scattering problem, Lett. Math. Phys., 7 (1983), 517-521.

[13] Devchand, C., An infinite number of continuity equations and hidden symmetries in supersymmetric gauge theories, Nucl. Phys., B238, (1984), 33-348.

[14] Chau, L.-L., Ge, M.-L. and Popowicz, Z., Riemann-Hilbert transforms and BianchiBäcklund iransformations for the supersymmetric Yang-Mills fields, Phys. Rev. Lett., 52 (1984), 1940-1943.

[15] Ward, R. S., On the self-dual gauge fields, Phys. Lett., 611 (1977), 81-82.

[16] Penrose, R., Nonlinear graviton and curved twistor theory, Gen. Rel. Grav., 7 (1976), 31-52.

[17] Boyer, C. P., The geometry of complex self-dual Einstein spaces, in ref. [3].

[18] Boyer, C. P. and Plebanski, J. F., An infinite hierarchy of conservation laws and superposition principles for self-dual Einstein spaces, J. Math. Phys., 26 (1985), 229-234.

[19] Eguch, T., Gilkey, P. B. and Hanson, A. J., Gravitation, gauge theories and differential geometry, Phys. Pep., 66 (1980), 213-293.

[20] Plebanski, J. F., Some solutions of comlex Einstein equations, J. Math. Phys., 16 (1975), 2395-2402.

[21] Newman, E. T., Porter, J. R. and Tod, K. P., Twistor surfaces and right-flat spaces, Gen. Rel. Grav., 9 (1978), 1129-1142.

[22] Sternberg, S., Lectures on differential geometry, Eaglewood Cliffs, Prentice Hall, New York, 1964.

[23] Estabrook, H. D. and Wahlquist, N. J., Prolongation structures of nonlinear evolution equations, J. Math. Phys., 16 (1975), 1-7.

[24] Atiyah, M. F., Hitchin, N. J., and Singer, I. M., Self-duality in four dimensional Riemannian geometry, Proc. R. Soc. London, A362 (1978), 425-461.

[25] Torres del Castillo, G. F., Gauge fields in algebraically special space-times, J. Math. Phys., 26 (1985), 836-840.

[26] See refs. [2, 3, 7, 10, 14] and references cited therein.

[27] Curtis, W. D., Lerner, D. E. and Miller, F. R., Complex pp waves and the nonlinear graviton construction, J. Math. Phys., 19 (1978), 2024-2027.

[28] Ward, R. S., A class of self-dual solutions of Einstein's equations, Proc. R. Soc. London, A363 (1078), 289-295.

[29] Tod, K. P. and Ward, R. S., Self-dual metrics with self-dual Killing vectors, Proc. R. Soc. London, $\mathbb{A} 368$ (1979), 411-427. 
[30] Hitchin, N. J., Polygons and gravitons, Math. Proc. Cambridge Phil. Soc., 85 (1979), $465-476$.

- Twistor construction of Einstein metrics, in "Global Riemannian Geometry", ed. T. J. Wilmore and N. J. Hitchin, John Willey \& Sons 1984.

[31] Pommaret, J.F., Systems of partial differential equations and Lie pseudo-groups, Gordon and Breach, New York, 1978.

[32] Sato, M. and Sato, Y., Soliton equations as dynamical systems in infinite dimensional Grassmann manifold, in "Nonlinear P.D.E. in Applied Science", U.S.-Japan seminor Tokyo 1982, ed. P. D. Lax and H. Fujita, North-Holland 1982.

[33] Takasaki, K., A new approach to the self-dual Yang-Mills equations, Commun. Math. Phys., 94 (1984), 35-59.

- ditto II, Saitama Math. J., 3 (1985), 11-40.

[34] Suzuki, N., Structure of the solution space of Witten's gauge-field equations, Proc. Japan Acad., 60A (1984), 141-144.

[35] Harnad, J. and Jacques, M., Formal power series solutions of supersymmetric $(\mathrm{N}=3)$ Yang-Mills equations, Preprint.

[36] Sanchéz, N., Einstein equations without Killing vectors, nonlinear sigma models, and self-dual Yang-Mills theory, in "Differential Geometric Methods in Theoretical Physics", ed. G. Denardo, G. Chirardi and T. Weber, Lect. Notes. Phys., 193, Springer-Verlag 1984.

—, Einstein equations, self-dual Yang-Mills fields and nonlinear sigma models, Preprint.

[37] Gindikin, S. G., Integral geometry and twistors, in "Twistor geometry and Non-linear Systems", ed. H. D. Doebner and T. D. Palev, Lect. Notes. Math., 970, SpringerVerlag 1982.

-, Reduction of manifolds of rational curves and related problems of the theory of differential equations, Funct. Anal. Appl., 18 (1985), 278-298. 